\title{
ON DOMINANCY OF MINIMAL ACTIONS OF COMPACT KAC ALGEBRAS AND CERTAIN AUTOMORPHISMS IN $\operatorname{Aut}\left(\mathscr{A} / \mathscr{A}^{\alpha}\right)$
}

\author{
TAKEHIKO YAMANOUCHI
}

\begin{abstract}
We show that any minimal action of a compact Kac algebra with properly infinite fixed-point algebra is dominant. It is also shown that, for such a minimal action, a certain automorphism in $\operatorname{Aut}\left(\mathscr{A} / \mathscr{A}^{\alpha}\right)$ gives rise to a unitary that lies in the intrinsic group of the dual Kac algebra. A concrete description, in terms of the unitary, of the left co-ideal von Neumann subalgebra determined by the fixed-point algebra of the automorphism is given.
\end{abstract}

\section{Introduction}

In the classification of group actions on (AFD) von Neumann algebras, the case of compact groups has long occupied a central position. One immediately realizes that the situation is already complicated enough and hard to be analyzed even when groups in question are finite ones. However, the classification of them, up to (cocycle) conjugacy, was successfully completed by Jones [J1] after the Connes' breakthrough in [C]. In the infinite case, abelian groups have been first intensively studied by several mathematicians $[\mathrm{J} 2],[\mathrm{OPT}],[\mathrm{JT}],[\mathrm{T}],[\mathrm{KT}]$, etc., and it is completed in [KT]. To the contrary, nonabelian case is far from being completed. To the best of author's knowledge, only partial results have been obtained so far, such as for ergodic actions [W1, 2, 3], and for minimal actions [PW].

In connection with this classification, Izumi-Longo-Popa have recently succeeded in establishing the complete Galois correspondence of minimal actions of compact groups as well as those of compact Kac algebras [ILP], which has been a long-standing problem since 70's (c.f. [NT]). Although we are at this point short of concrete intriguing examples of such actions, we believe that their result is important enough to ensure that minimal actions of compact Kac algebras deserve a further investigation. Thus we pursue this line of research here.

Received January 27, 1997. 
Our aim of this article is (i) to show that every minimal action of a compact Kac algebra is dominant (see Definition 2.1 below); (ii) to prove that, for a minimal action $\alpha$, a certain automorphism in $\operatorname{Aut}\left(\mathscr{A} / \mathscr{A}^{\alpha}\right)$ gives rise to a group-like element of the dual Kac algebra.

The organization of the paper is as follows. In Section 1, we introduce terminologies and notation used in the following sections. In Section 2, we show that any minimal action of a compact Kac algebra on a factor with properly infinite fixed-point algebra is dominant, hence dual in particular. Part of this statement, i.e., occurrence of every irreducible representation of the Kac algebra in the given minimal action, is referred in the proof of Theorem 4.4 of [ILP]. We give a complete proof to it in Proposition 2.14. In Section 3, we prove that, given such a minimal action $\alpha$ of a compact Kac algebra $\mathrm{K}$ on a factor $\mathscr{A}$, an automorphism $\theta$ in $\operatorname{Aut}\left(\mathscr{A} / \mathscr{A}^{\alpha}\right)$ leaving each of the $\alpha$-invariant Hilbert spaces in $\mathscr{A}$ (see Section 2 ) globally invariant gives rise to a unitary $u(\theta)$ in the intrinsic group of the dual Kac algebra. Moreover, we show that the left-coideal von Neumann subalgebra $\mathscr{N}(\theta)$ determined by the intermediate subfactor $\mathscr{A}^{\theta}$, according to the Galois correspondence of [ILP], is concretely described in terms of the unitary $u(\theta)$. Finally, in Appendix, we collect some general results on actions of Kac algebras on von Neumann algebras that are needed in Section 2-3. We believe that almost all results stated there are well-known to specialists. But the author was unable to locate a literature that actually contains them. So we provide their proofs for completeness of our argument.

\section{Terminology and Notation}

In this section, we briefly review fundamental results on (compact) Kac algebras, and introduce notation which will be necessary for our discussion that follows. For the general theory of Kac algebras, we refer to [ES1], the notation of which we mainly adopt as well.

From now on, all von Neumann algebras are assumed to have separable preduals.

A Kac algebra is a quadruple $\mathrm{K}=(\mathscr{M}, \Gamma, \kappa, \varphi)$ [ES1, Definition 2.2.5] in which:

(Ki) $(\mathscr{M}, \Gamma, \kappa)$ is a co-involutive Hopf von Neumann algebra [ES1, Definition 1.2.5];

(Kii) $\varphi$ is a faithful normal semifinite weight on $\mathscr{M}$, called a Haar measure (weight) of $\mathrm{K}$;

(Kiii) $\left(\right.$ id $\left._{\mathscr{M}} \otimes \varphi\right) \Gamma(x)=\varphi(x) \cdot 1 \quad\left(x \in \mathscr{M}_{+}\right)$;

(Kiv) $\left(i d_{\mathscr{M}} \otimes \varphi\right)\left(\left(1 \otimes y^{*}\right) \Gamma(x)\right)=\kappa\left(\left(i_{\mathscr{M}} \otimes \varphi\right)\left(\Gamma\left(y^{*}\right)(1 \otimes x)\right)\right)$

$\left(x, y \in N_{\varphi}\right)$; 
$(\mathrm{Kv}) \kappa \circ \sigma_{t}^{\varphi}=\sigma_{-t}^{\varphi} \circ \kappa \quad(t \in \mathrm{R})$.

We say that $\mathrm{K}$ is compact if $\varphi(1)<\infty$. In this case, it turns out that $\varphi$ is a trace with $\varphi \circ \kappa=\varphi$. Let us fix a Kac algebra $\mathrm{K}=(\mathscr{M}, \Gamma, \kappa, \varphi)$. We always think of $\mathscr{M}$ as represented on the Hilbert space $L^{2}(\varphi)$ obtained from $\varphi$. Let $\Lambda_{\varphi}$ denote the canonical injection of $\mathscr{M}$ into $L^{2}(\varphi)$. Then the equation

$$
W\left(\Lambda_{\varphi}(x) \otimes \Lambda_{\varphi}(y)\right)=\Lambda_{\varphi \otimes \varphi}(\Gamma(y)(x \otimes 1)) \quad\left(x, y \in N_{\varphi}\right)
$$

defines a unitary on $L^{2}(\varphi) \otimes L^{2}(\varphi)$, called the fundamental unitary of $\mathrm{K}$ [ES1, Proposition 2.4.2], and denoted by $W(\mathrm{~K})$ if an unnecessary confusion may occur. It implements $\Gamma: \Gamma(x)=W(1 \otimes x) W^{*}(x \in \mathscr{M})$. The set (group) $G(\mathrm{~K})$ of unitaries $u$ in $\mathscr{M}$ satisfying $\Gamma(u)=u \otimes u$ is called the intrinsic group of $\mathrm{K}[\mathrm{DeC}],[\mathrm{S}]$.

The main feature of the theory is the construction of the dual Kac algebra $\hat{\mathrm{K}}=(\hat{\mathscr{M}}, \hat{\Gamma}, \hat{\kappa}, \hat{\varphi})$ [ES1, Chap 3]. The fundamental unitary $W(\hat{\mathrm{K}})$ of $\hat{\mathrm{K}}$ is $\Sigma W(\mathrm{~K})^{*} \Sigma$ [ES1, Theorem 3.7.3], where $\Sigma$ in general stands for the flip (twist) operator: $\Sigma(\xi \otimes \eta)=\eta \otimes \xi$. There are another Kac algebras canonically attached to $\mathrm{K}$, such as $\mathrm{K}^{\prime}=$ the commutant of $\mathrm{K}, \mathrm{K}^{\sigma}=$ the opposite of $\mathrm{K}$, etc. (see [ES1]).

The predual $\mathscr{M}_{*}$ becomes an involutive Banach algebra. We shall be mainly concerned with (nondegenerate) representations of $\mathscr{M}_{*}$. By [ES1, Theorem 3.1.4], any representation $\mu$ of $\mathscr{M}_{*}$ on a Hilbert space $\mathscr{H}_{\mu}$ admits a generator, i.e., there is a unitary $V$ on $\mathscr{H}_{\mu} \otimes L^{2}(\varphi)$ such that $\mu(\omega)=(\mathrm{id} \otimes \omega)(V)$. The representation $\lambda$ that has $W(\hat{\mathrm{K}})$ as a generator is called the regular (Fourier) representation of $\mathrm{K}$. It generates the dual Kac algebra $\hat{\mathscr{M}}$.

An action of $\mathrm{K}$ on a von Neumann algebra $\mathscr{A}$ [E] is a unital injective *-homomorphism $\alpha$ from $\mathscr{A}$ into $\mathscr{A} \otimes \mathscr{M}$ satisfying $\left(\alpha \otimes \mathrm{id}_{\mathscr{M}}\right) \circ \alpha=$ $\left(\mathrm{id}_{\mathscr{A}} \otimes \Gamma\right) \circ \alpha$. Conjugacy and cocycle conjugacy of actions are defined as in the case of group actions (see [E], [N]). The crossed product $\mathscr{A} \rtimes_{\alpha} \mathrm{K}$ of $\mathscr{A}$ by the action $\alpha$ is by definition the von Neumann algebra generated by $\alpha(\mathscr{A})$ and $\mathbf{C} \otimes \hat{\mathscr{M}}^{\prime}$. On the crossed product, there exists an action $\hat{\alpha}$ of $\hat{\mathrm{K}}^{\prime}$, called the dual action of $\alpha$ [E, Définition II.7 ]. In the meantime, there is an action $\bar{\alpha}$ of $\mathrm{K}$ on $\mathscr{A} \otimes \mathscr{L}\left(L^{2}(\varphi)\right)$ (or, more generally, on $\mathscr{A} \otimes \mathscr{B}$ with $\mathscr{B}$ another von Neumann algebra), defined by $\bar{\alpha}=\left(\operatorname{id}_{\mathscr{A}} \otimes \sigma\right) \circ(\alpha \otimes \mathrm{id})$, where $\sigma=\operatorname{Ad} \Sigma$. We call $\bar{\alpha}$ the amplified action of $\alpha$. On $\mathscr{A} \otimes \mathscr{L}\left(L^{2}(\varphi)\right)$, there is another action $\tilde{\alpha}$ of $\mathrm{K}$ defined by $\tilde{\alpha}=\operatorname{Ad}(1 \otimes W(\hat{\mathrm{K}})) \circ \bar{\alpha}$. It is known [ES2, Proposition IV.5] that the bidual action $\hat{\alpha}$ is conjugate to $\tilde{\alpha}$. The fixed-point algebra $\mathscr{A}^{\alpha}$ of $\alpha$ is defined to be the set $\{x \in \mathscr{A}: \alpha(x)=x \otimes 1\}$.

Finally, theory of compact Kac algebras almost goes parallel to that of compact groups [ES1, §6]. For example, the Peter-Weyl theorem, the Schur's 
orthogonality relations, complete reducibility, etc. hold true [ES1, Rheorem 6.2.5 and 6.2.6]. See also [W].

\section{Dominancy of minimal actions}

This section is devoted to proving the first main theorem of this note on dominancy of minimal actions of compact Kac algebras. In what follows, we shall retain the notation established in the preceding section. Let us fix a Kac algebra $\mathrm{K}=(\mathscr{M}, \Gamma, \kappa, \varphi)$ once and for all.

Definition 2.1. Let $\alpha$ be an action of $\mathrm{K}$ on a von Neumann algebras $\mathscr{A}$.

(1) (Minimality: see [ILP]) We say that $\alpha$ is minimal if

(i) the fixed-point algebra $\mathscr{A}^{\alpha}$ of $\alpha$ has the trivial relative commutant in $\mathscr{A}$, i.e., $\left(\mathscr{A}^{\alpha}\right)^{\prime} \cap \mathscr{A}=\mathrm{C}$;

(ii) the linear span of the set $\left\{\left(\omega \otimes \mathrm{id}_{\mathscr{M}}\right) \circ \alpha(x): \omega \in \mathscr{A}_{*}, x \in \mathscr{A}\right\}$ is $\sigma$ weakly dense in $\mathscr{M}$.

(2) (Dominancy: c.f. [CT], [NT]) The action $\alpha$ is said to be dominant if

(i) $\mathscr{A}^{\alpha}$ is properly infinite;

(ii) $\alpha$ is dual, that is, the dual action of an action on some von Neumann algebra (which is necessarily isomorphic to $\mathscr{A}^{\alpha}$ ).

With these definitions, we can state our main theorem.

TheORem 2.2. Suppose that $\mathrm{K}$ is a compact Kac algebra. If $\alpha$ is a minimal action of $\mathrm{K}$ on a factor $\mathscr{A}$ with properly infinite fixed-point algebra, then it is dominant.

To prove this theorem, we require a series of preparatory results. Our approach to the proof is, however, basically quite the same as that of group action case. Thus it will be our central goal to show that every irreducible representation of $\mathscr{M}_{*}$ is unitarily equivalent to "the subrepresentation obtained by restricting the action $\alpha$ to some $\alpha$-invariant Hilbert space in $\mathscr{A}$." This can be described in Roberts' terminology [R] by saying that $\alpha$ has full monoidal spectrum.

In the discussion that follows, we assume that $\mathrm{K}$ is a compact $\mathrm{Kac}$ algebra with the normalized Haar measure $\varphi$. We also fix an action $\alpha$ of $\mathrm{K}$ on a von Neumann algebra $\mathscr{A}$. It is well-known (see [ES2, Proposition II.2]) that $E_{\alpha}=(\mathrm{id} \otimes \varphi) \circ \alpha: \mathscr{A} \rightarrow \mathscr{A}$ is a faithful normal conditional expectation from $\mathscr{A}$ onto the fixed-point algebra $\mathscr{A}^{\alpha}$. Take a faithful normal state $\omega_{0}$ on $\mathscr{A}^{\alpha}$. Then set $\psi=\omega_{0} \circ E_{\alpha}$. From now on, we shall always think of $\mathscr{A}$ as represented on $L^{2}(\psi)$, the Hilbert space obtained from $\psi$ by the GNS construction.

For each irreducible representation $\left(\pi, \mathscr{H}_{\pi}\right)$ of $\mathscr{M}_{*}$ with generator $V(\pi) \in \mathscr{L}\left(\mathscr{H}_{\pi}\right) \otimes \mathscr{M}$, we define the $\pi$-eigenspace $\mathscr{A}^{\alpha}(\pi)$ to be the set given by 
ON DOMINANCY OF MINIMAL ACTIONS OF COMPACT KAC ALGEBRAS... 301

$$
\mathscr{A}^{\alpha}(\pi)=\left\{X \in \mathscr{A} \otimes \mathscr{L}\left(\mathscr{H}_{\pi}\right): \bar{\alpha}(X)=X_{12} V(\pi)_{23}\right\},
$$

where, in this case, $\bar{\alpha}$ is the amplified action $\bar{\alpha}=\left(\operatorname{id}_{\mathscr{A}} \otimes \sigma\right) \circ\left(\alpha \otimes \mathrm{id}_{\mathscr{L}\left(\mathscr{H}_{\pi}\right)}\right)$. Any element in $\mathscr{A}^{\alpha}(\pi)$ is called a $\pi$ - eigenoperator. As in the case of compact group actions, these eigenspaces $\left\{\mathscr{A}^{\alpha}(\pi)\right\}_{\pi \in \operatorname{Irr}\left(\mathscr{M}_{*}\right)}$ shall play a crucial part in our study.

A finite-dimensional subspace $D$ of $\mathscr{A}$ is said to be $\alpha$-invariant if $\alpha(D) \subseteq D \otimes \mathscr{M}$. If $D$ is $\alpha$-invariant, then it determines an operator $V \in \operatorname{End}(D) \otimes \mathscr{M} \quad$ satisfying $\quad V_{12} V_{13}=($ id $\otimes \Gamma)(V)$. In fact, with $\left\{v_{1}, v_{2}, \ldots, v_{d}\right\}$ a basis for $D$, the equation

$$
\alpha\left(v_{j}\right)=\sum_{i=1}^{d} v_{i} \otimes V_{i, j} \quad(j=1,2, \ldots, d)
$$

determines a family $\left\{V_{i, j}\right\}$ of elements in $\mathscr{M}$. Then put

$$
V=\sum_{i, j=1}^{d} e_{i, j} \otimes V_{i, j} \in \operatorname{End}(D) \otimes \mathscr{M},
$$

where $\left\{e_{i, j}\right\}$ denotes the system of matrix units in $\operatorname{End}(D)$, corresponding to the basis $\left\{v_{i}\right\}$. It is easily verified that $V$ is independent of the choice of the basis $\left\{v_{i}\right\}$, and that it satisfies $V_{12} V_{13}=(\mathrm{id} \otimes \Gamma)(V)$. From the last identity, it follows that the equation

$$
\pi(\omega)=(\operatorname{id} \otimes \omega)(V) \quad\left(\omega \in \mathscr{M}_{*}\right)
$$

defines a (not necessarily $*$-preserving) homomorphism $\pi$ of $\mathscr{M}_{*}$ into $\operatorname{End}(D)$. We call the pair $(\pi, V)$ constructed in this way the representation associated with the $\alpha$-invariant subspace $D$, and denote it by $\left(\pi_{D}, V(D)\right)$. From (2.3), we immediately see that

$$
\pi_{D}(\omega) v=(\mathrm{id} \otimes \omega) \circ \alpha(v) \quad(v \in D) .
$$

The following lemma is a Kac algebraic version of Proposition 2.2 of [NT, Chap. IV], for which we do not need to assume minimality of $\alpha$ or $\mathscr{A}^{\alpha}$ being properly infinite.

Lemma 2.6. Let $\left(\pi, \mathscr{H}_{\pi}\right)$ be an irreducible representation of $\mathscr{M}_{*}$. Then the following are equivalent:

(i) $\mathscr{A}^{\alpha}(\pi)$ contains a non-zero element;

(ii) there exists an $\alpha$-invariant subspace $D$ so that $\pi_{D}$ is equivalent to $\pi$, in the sense that there is an invertible linear operator $T: \mathscr{H}_{\pi} \longrightarrow D$ satisfying $(T \otimes 1) V(\pi)=V(D)(T \otimes 1)$, or equivalently, $T \pi(\omega) T^{-1}=\pi_{D}(\omega) \quad\left(\omega \in \mathscr{M}_{*}\right)$. 
Proof. (i) $\Rightarrow$ (ii). Take a non-zero $X \in \mathscr{A}^{\alpha}(\pi)$. With $\left\{\varepsilon_{i}\right\}_{i=1}^{d=\operatorname{dim} \mathscr{H}_{\pi}}$ an ONB (orthonormal basis) for $\mathscr{H}_{\pi}$, and $\left\{e_{i, j}\right\}$ the corresponding matrix units of $\mathscr{L}\left(\mathscr{H}_{\pi}\right)$, we write $X$ and $V(\pi)$ in the form

$$
X=\sum_{j, k=1}^{d} X_{j, k} \otimes e_{j, k}, \quad V(\pi)=\sum_{j, k=1}^{d} e_{j, k} \otimes V(\pi)_{j, k} .
$$

Note that, with the notation as above, the identity $\bar{\alpha}(X)=X_{12} V(\pi)_{23}$ is equivalent to

$$
\alpha\left(X_{j, k}\right)=\sum_{i=1}^{d} X_{j, i} \otimes V(\pi)_{i, k} \quad(1 \leq j, k \leq d) .
$$

This fact will be often used in the later discussion. Since $X \neq 0$, there are $j_{0}, k_{0}$ such that $X_{j_{0}, k_{0}} \neq 0$. With $v_{k}=X_{j_{0}, k}(k=1,2, \ldots, d)$, we define $D$ to be the linear span of $\left\{v_{k}\right\}$. By (2.7), $D$ is $\alpha$-invariant. Moreover, from (2.5) and (2.7), it follows that, for $\omega \in \mathscr{M}_{*}$, we have

$$
\pi_{D}(\omega) v_{j}=(\operatorname{id} \otimes \omega) \circ \alpha\left(v_{j}\right)=\sum_{i=1}^{d} \omega\left(V(\pi)_{i, j}\right) v_{i} .
$$

Thus, if we define a linear operator $T: \mathscr{H}_{\pi} \longrightarrow D$ by $T \varepsilon_{i}=v_{i}$, then we obtain $\pi_{D}(\omega) T=T \pi(\omega)$. Since $\pi$ is irreducible and $D$ is a non-zero vector space, $T$ is invertible.

(ii) $\Rightarrow$ (ii). A proof can be obtained by tracing back the preceding paragraph. With $\left\{\varepsilon_{i}\right\}$ as in (i) $\Rightarrow$ (ii), set $v_{i}=T \varepsilon_{i}(i=1,2, \ldots, d)$. If $e_{i, j}^{\prime}$ denotes the matrix units of $\operatorname{End}(D)$ determined by the basis $\left\{v_{i}\right\}$, then the identity $(T \otimes 1) V(\pi)=V(D)(T \otimes 1)$ yields $V(D)=\sum_{i, j=1}^{d} e_{i, j}^{\prime} \otimes V(\pi)_{i, j}$. Thus we have $\alpha\left(v_{j}\right)=\sum_{i=1}^{d} v_{i} \otimes V(\pi)_{i, j}$ (see (2.3) and (2.4)). So, with $X_{j, k}=v_{k}$ $(1 \leq j, k \leq d)$, we get equation (2.7). Therefore, $X=\left[X_{j, k}\right] \in \mathscr{A} \otimes \mathscr{L}\left(\mathscr{H}_{\pi}\right)$ is a $\pi$-eigenoperator. Clearly, $X$ is non-zero.

Lemma 2.8. (c.f. [R], [AKHT], [NT]). Let $\left(\pi, \mathscr{H}_{\pi}\right)$ be an irreducible representation of $\mathscr{M}_{*}$. Suppose that $\mathscr{A}^{\alpha}$ is properly infinite. Then the following are equivalent:

(i) $\mathscr{A}^{\alpha}(\pi)$ contains a unitary;

(ii) there exists an $\alpha$-invariant Hilbert space $D$ in $\mathscr{A}$ such that $\pi_{D}$ is unitarily equivalent to $\pi$.

Proof. With $d=\operatorname{dim} \mathscr{H}_{\pi}$, let $\left\{\varepsilon_{i}\right\}_{i=1}^{d}$ be an ONB for $\mathscr{H}_{\pi}$ and $\left\{e_{i, j}\right\}$ the corresponding matrix units of $\mathscr{L}\left(\mathscr{H}_{\pi}\right)$.

(i) $\Rightarrow$ (ii). Take a unitary $X=\sum_{i=1}^{d} X_{i, j} \otimes e_{i, j} \in \mathscr{A}^{\alpha}(\pi)$. Since $\mathscr{A}^{\alpha}$ is prop- 
erly infinite, there is a Hilbert space $K$ in $\mathscr{A}^{\alpha}$ with $\operatorname{dim} K=d$. Let $\left\{w_{i}\right\}_{i=1}^{d}$ be an ONB for $K$. Then define a unitary $V$ from $L^{2}(\psi)$ onto $L^{2}(\psi) \otimes \mathscr{H}_{\pi}$ by

$$
V \xi=\sum_{i=1}^{d} w_{i}^{*} \xi \otimes \varepsilon_{i} \quad\left(\xi \in L^{2}(\psi)\right) .
$$

The inverse $V^{-1}=V^{*}$ is given by $V^{-1}\left(\sum_{i=1}^{d} \eta_{i} \otimes \varepsilon_{i}\right)=\sum_{i=1}^{d} w_{i} \eta_{i}$. We put $v_{i}=V^{*} X V w_{i} \in \mathscr{L}\left(L^{2}(\psi)\right) \quad(i=1,2, \ldots, d)$. Since $\quad v_{i}^{*} v_{j}=\delta_{i, j} \cdot 1 \quad$ and $\sum_{i=1}^{d} v_{i} v_{i}^{*}=1$, the linear span $D$ of $\left\{v_{i}\right\}$ forms a Hilbert space in $\mathscr{L}\left(L^{2}(\psi)\right)$. For any $\xi \in L^{2}(\psi)$, we have

$$
v_{i} \xi=V^{*} X V w_{i} \xi=V^{*} X\left(\xi \otimes \varepsilon_{i}\right)=V^{*}\left(\sum_{j=1}^{d} X_{j, i} \xi \otimes \varepsilon_{j}\right)=\sum_{j=1}^{d} w_{j} X_{j, i} \xi .
$$

This shows that $v_{j}=\sum_{i=1}^{d} w_{i} X_{i, j} \in \mathscr{A}$, so that $D$ is a Hilbert space in $\mathscr{A}$. Since $X_{j, k}$ satisfies (2.7) and $w_{i}$ belongs to $\mathscr{A}^{\alpha}$, we have

$$
\alpha\left(v_{j}\right)=\sum_{i=1}^{d} v_{i} \otimes V(\pi)_{i, j} \quad(j=1,2, \ldots, d) .
$$

From this, it follows easily that $D$ is $\alpha$-invariant, and that, with a unitary $U: \mathscr{H}_{\pi} \rightarrow D$ defined by $U \varepsilon_{i}=v_{i}$, one has $(U \otimes 1) V(D)=V(\pi)(U \otimes 1)$ and $\pi_{D}(\omega) U=U \pi(\omega)$ for any $\omega \in \mathscr{M}_{*}$.

(ii) $\Rightarrow$ (i). Let $K,\left\{w_{i}\right\}$ and $V$ be as in the preceding paragraph. By assumption, we may choose an ONB $\left\{v_{i}\right\}$ of $D$ so that (2.9) holds. Then define an operator $\quad X \in \mathscr{L}\left(L^{2}(\psi)\right) \otimes \mathscr{L}\left(\mathscr{H}_{\pi}\right) \quad$ by $\quad X=\sum_{i=1}^{d} V v_{i} w_{i}^{*} V^{*}$. Since $V y=(y \otimes 1) V$ for any $y \in \mathscr{A}^{\prime}$. It follows that $X \in \mathscr{A} \otimes \mathscr{L}\left(\mathscr{H}_{\pi}\right)$. Simple calculations show that $X$ is a unitary. Moreover, we have

$$
X\left(\xi \otimes \varepsilon_{j}\right)=\sum_{k=1}^{d} w_{k}^{*} v_{j} \xi \otimes \varepsilon_{k}
$$

Thus

$$
X=\sum_{i, j=1}^{d} w_{i}^{*} v_{j} \otimes e_{i, j}
$$

From (2.9) and the fact that $w_{i} \in \mathscr{A}^{\alpha}$, it results that the matrix components $\left\{X_{i, j}:=w_{i}^{*} v_{j}\right\}$ of $X$ satisfies (2.7). Therefore, $X$ is an $\pi$-eigenoperator.

For each irreducible representation $\left(\pi, \mathscr{H}_{\pi}\right)$, we define a map $\beta_{\pi}$ from $\mathscr{A} \otimes \mathscr{L}\left(\mathscr{H}_{\pi}\right)$ into $\mathscr{A} \otimes \mathscr{L}\left(\mathscr{H}_{\pi}\right) \otimes \mathscr{M}$ by

$$
\beta_{\pi}=\operatorname{Ad}(1 \otimes V(\pi)) \circ \bar{\alpha},
$$


where, as usual, $\bar{\alpha}$ is the amplified action: $\bar{\alpha}=\left(\operatorname{id}_{\mathscr{A}} \otimes \sigma\right) \circ\left(\alpha \otimes \mathrm{id}_{\mathscr{L}\left(\mathscr{H}_{\pi}\right)}\right)$. The map $\beta_{\pi}$ is an action of $\mathrm{K}$ on $\mathscr{Q}=\mathscr{A} \otimes \mathscr{L}\left(\mathscr{H}_{\pi}\right)$. One can verify this by a direct computation, but another way to do that is to notice that $\beta_{\pi}$ can be written in the form: $\beta_{\pi}=\alpha *_{V(\pi)} \gamma_{\pi}$, where $\gamma_{\pi}$ is an action of $\mathrm{K}$ on $\mathscr{L}\left(\mathscr{H}_{\pi}\right)$ defined by $\gamma_{\pi}(z)=V(\pi)(z \otimes 1) V(\pi)^{*}\left(z \in \mathscr{L}\left(\mathscr{H}_{\pi}\right)\right)$ (recall that $V(\pi)$ is an $I_{\mathrm{K}}^{\mathscr{L}\left(\mathscr{H}_{\pi}\right)}$-cocycle), and $\alpha *_{V(\pi)} \gamma_{\pi}$ stands for the inner tensor product action of $\alpha$ and $\gamma_{\pi}$ in the sense of Nakagami (see $[\mathrm{N}, \S 5,(5.2)]$ ). The following lemma is a simple exercise, so it is left to readers.

Lemma 2.10. The $\pi$-eigenspace $\mathscr{A}^{\alpha}(\pi)$ is a $\mathscr{Q}^{\bar{\alpha}}$ - $\mathscr{Q}^{\beta_{\pi}}$ bimodule.

Lemma 2.11. Suppose that the relative commutant of $\mathscr{A}^{\alpha}$ in $\mathscr{A}$ is trivial. Then both the left and the right support projections of $\mathscr{A}^{\alpha}(\pi)$ are equal to the identity operator 1 for any $\pi$ with $\mathscr{A}^{\alpha}(\pi) \neq\{0\}$.

Proof. Let $e_{1}$ and $e_{2}$ be the left and the right support projection of $\mathscr{A}^{\alpha}(\pi)$, respectively. Suppose that $\mathscr{A}^{\alpha}(\pi) \neq\{0\}$.

First, we show that $\mathscr{A}^{\alpha}(\pi)$ is stable under the adjoint action of the unitary group $\mathscr{U}\left(\mathscr{A}^{\alpha}\right)$ of $\mathscr{A}^{\alpha}$. So set $\delta_{u}=\operatorname{Ad} u\left(u \in \mathscr{U}\left(\mathscr{A}^{\alpha}\right)\right)$. It is apparant that we have $\alpha \circ \delta_{u}=\left(\delta_{u} \otimes \operatorname{id}_{\mathscr{M}}\right) \circ \alpha$. Let $\bar{\delta}_{u}=\delta_{u} \otimes \operatorname{id}_{\mathscr{L}\left(\mathscr{H}_{\pi}\right)}$. Then, with $X \in \mathscr{A}^{\alpha}(\pi)$, we have

$$
\begin{aligned}
\bar{\alpha} \circ \bar{\delta}_{u}(X) & =\left(\operatorname{id}_{\mathscr{A}} \otimes \sigma\right) \circ\left(\alpha \circ \delta_{u} \otimes \operatorname{id}_{\mathscr{L}\left(\mathscr{H}_{\pi}\right)}\right)(X) \\
& =\left(\operatorname{id}_{\mathscr{A}} \otimes \sigma\right) \circ\left(\left(\delta_{u} \otimes \operatorname{id}_{\mathscr{M}}\right) \circ \alpha \otimes \operatorname{id}_{\mathscr{L}\left(\mathscr{H}_{\pi}\right)}\right)(X) \\
& =\left(\delta_{u} \otimes \operatorname{id}_{\mathscr{L}\left(\mathscr{H}_{\pi}\right)} \otimes \operatorname{id}_{\mathscr{M}}\right) \circ \bar{\alpha}(X)=\bar{\delta}_{u}(X)_{12} V(\pi)_{23} .
\end{aligned}
$$

This proves that $\bar{\delta}_{u}\left(\mathscr{A}^{\alpha}(\pi)\right)=\mathscr{A}^{\alpha}(\pi)$. From this, it follows that $\bar{\delta}_{u}\left(e_{i}\right)=e_{i}$ for any $u \in \mathscr{U}\left(\mathscr{A}^{\alpha}\right) \quad(i=1,2)$. By our hypothesis, we have $2^{\bar{\delta}}=\mathscr{A}^{\delta} \otimes \mathscr{L}\left(\mathscr{H}_{\pi}\right)=\otimes \mathscr{L}\left(\mathscr{H}_{\pi}\right)$. Hence $e_{i}=1 \otimes q_{i}$ for some projections $q_{i} \in \mathscr{L}\left(\mathscr{H}_{\pi}\right)$.

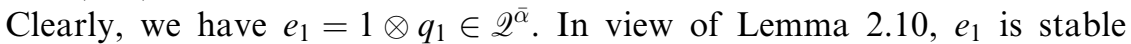
under the adjoint action of the unitary group of $2^{\bar{\alpha}}$. Hence $e_{1}$ belongs to the center of $2^{\bar{\alpha}}$, which is the scalar multiples of the identity by assumption. Thus $e_{1}=1$.

It remains to show that $q_{2}=1$. For any $X \in \mathscr{A}^{\alpha}(\pi)$, since $\bar{\alpha}(X)=X_{12} V(\pi)_{23}$, we have $\bar{\alpha}\left(X^{*} X\right)=V(\pi)_{23}^{*}\left(X^{*} X\right)_{12} V(\pi)_{23}$, so that $\beta_{\pi}\left(X^{*} X\right)=\left(X^{*} X\right)_{12}$. Namely, $X^{*} X$ belongs to $2^{\beta_{\pi}}$, and so does the right support of $X$. Hence $e_{2} \in 2^{\beta_{\pi}}$. From this, it follows that

$$
1 \otimes q_{2} \otimes 1=e_{2} \otimes 1=\beta_{\pi}\left(e_{2}\right)=(1 \otimes V(\pi))\left(1 \otimes q_{2} \otimes 1\right)\left(1 \otimes V(\pi)^{*}\right) .
$$

Thus $q_{2} \otimes 1=V(\pi)\left(q_{2} \otimes 1\right) V(\pi)^{*}$. Since $\pi$ is irreducible, $q_{2}=1$.

Let us now prove a key proposition to the proof of Theorem 2.3. 
Proposition 2.12. Suppose that the fixed-point algebra $\mathscr{A}^{\alpha}$ is properly infinite and has the trivial relative commutant in $\mathscr{A}$. Then, for any irreducible representation $\left(\pi, \mathscr{H}_{\pi}\right), \mathscr{A}^{\alpha}(\pi) \neq\{0\}$ if and only if $\mathscr{A}^{\alpha}(\pi)$ contains a unitary.

Proof. First observe that $X^{*} Y \in \mathscr{2}^{\beta_{\pi}}$ and $X Y^{*} \in \mathscr{2}^{\bar{\alpha}}$ whenever $X, Y \in \mathscr{A}^{\alpha}(\pi)$. From this observation and Lemma 2.10, it follows that the set

$$
\mathscr{R}=\left\{\left[\begin{array}{cc}
a & X \\
Y^{*} & b
\end{array}\right]: a \in \mathscr{Q}^{\bar{\alpha}}, X, Y \in \mathscr{A}^{\alpha}(\pi), b \in \mathscr{Q}^{\beta_{\pi}}\right\}
$$

is a von Neumann subalgebra of $\mathscr{Q} \otimes M_{2}(\mathrm{C})$. With

$$
e_{1}=\left[\begin{array}{ll}
1 & 0 \\
0 & 0
\end{array}\right], \quad e_{2}=\left[\begin{array}{ll}
0 & 0 \\
0 & 1
\end{array}\right],
$$

we have

$$
\begin{aligned}
& e_{1} \mathscr{R} e_{1}=\left\{\left[\begin{array}{ll}
a & 0 \\
0 & 0
\end{array}\right]: a \in \mathscr{Q}^{\bar{\alpha}}\right\} \cong \mathscr{Q}^{\bar{\alpha}}=\mathscr{A}^{\alpha} \otimes \mathscr{L}\left(\mathscr{H}_{\pi}\right), \\
& e_{2} \mathscr{R} e_{2}=\left\{\left[\begin{array}{ll}
0 & 0 \\
0 & b
\end{array}\right]: b \in \mathscr{Q}^{\beta_{\pi}}\right\} \cong \mathscr{Q}^{\beta_{\pi}} \supseteq \mathscr{A}^{\alpha} \otimes \mathrm{C} .
\end{aligned}
$$

Since $\mathscr{2}^{\bar{\alpha}}$ and $\mathscr{2}^{\beta_{\pi}}$ are properly infinite, both $e_{1}$ and $e_{2}$ are properly infinite projections in $\mathscr{R}$. In the meantime,

$e_{1} \mathscr{R} e_{2}=\left\{\left[\begin{array}{cc}0 & X \\ 0 & 0\end{array}\right]: X \in \mathscr{A}^{\alpha}(\pi)\right\}, \quad e_{2} \mathscr{R} e_{1}=\left\{\left[\begin{array}{cc}0 & 0 \\ X^{*} & 0\end{array}\right]: X \in \mathscr{A}^{\alpha}(\pi)\right\}$.

From this, together with Lemma 2.11, we find that the left support projection of

$$
e_{1} \mathscr{R} e_{2}=\left[\begin{array}{cc}
\text { the left support projection of } \mathscr{A}^{\alpha}(\pi) & 0 \\
0 & 0
\end{array}\right]=e_{1} .
$$

Similarly, the left support projection of $e_{2} \mathscr{R} e_{1}$ coincides with $e_{2}$. These facts imply that the central supports of $e_{1}$ and $e_{2}$ are the same. Hence they are equivalent in $\mathscr{R}$. So there exists a matrix $v$

$$
v=\left[\begin{array}{cc}
a & X \\
Y^{*} & b
\end{array}\right] \in \mathscr{R}
$$

such that $v^{*} v=e_{1}$ and $v v^{*}=e_{2}$. This means that $Y$ is a unitary in $\mathscr{A}^{\alpha}(\pi)$.

LEMma 2.13. If $\alpha$ is an action satisfying the property (ii) in the definition of minimality (Definition 2.1 (1), (ii)), then $\mathscr{A}^{\alpha}(\pi) \neq\{0\}$ for any irreducible representation $\pi$. 
Proof. Suppose that there exists an irreducible representation $\pi$ such that the $\pi$-spectral subspace $\mathscr{A}_{\pi}$ (see Appendix, Part A) reduces to zero. Then, for any $a \in \mathscr{M}$ of the form $a=\left(\omega \otimes \operatorname{id}_{\mathscr{M}}\right) \circ \alpha(x) \quad\left(\omega \in \mathscr{A}_{*}, x \in \mathscr{A}\right)$, we have

$$
\varphi\left(a \chi_{\pi}^{*}\right)=\left(\omega \otimes \chi_{\pi}^{*} \varphi\right) \circ \alpha(x)=\omega\left(E_{\pi}(x)\right)=0,
$$

where $E_{\pi}$ is the map defined in Part A of Appendix. This implies by assumption that $\chi_{\pi}=0$, a contradiction.

Proposition 2.14. Suppose that $\alpha$ is a minimal action on $\mathscr{A}$ with properly infinite fixed-point algebra. Then $\mathscr{A}^{\alpha}(\pi)$ contains a unitary for every irreducible representation $\pi$. Therefore, there exists an $\alpha$-invariant Hilbert space $D$ in $\mathscr{A}$ such that $\pi_{D}$ is unitarily equivalent to $\pi$.

Proof. Let $\pi$ be an irreducible representation of $\mathscr{M}_{*}$. From Lemma 2.13, we have that $\mathscr{A}^{\alpha}(\pi) \neq\{0\}$. Then $\mathscr{A}^{\alpha}(\pi)$ contains a unitary by Propostion 2.12. The last assertion follows from Lemma 2.8.

With the aid of Proposition 2.14 just obtained above, we are now in a position to demonstrate a proof of Theorem 2.2.

Proof of Theorem 2.2. Let us denote by $\operatorname{Irr}=\operatorname{Irr}\left(\mathscr{M}_{*}\right)$ a complete set of representatives of the unitary equivalence classes of irreducible representations of $\mathscr{M}_{*}$.

Thanks to Proposition 2.14 , for each $\left(\pi, \mathscr{H}_{\pi}\right)$ in $\operatorname{Irr}\left(\mathscr{M}_{*}\right)$ with generator $V(\pi)$, we may choose a unitary $W(\pi)$ in $\mathscr{A}^{\alpha}(\pi)$. We set $W_{\alpha}=\sum_{\pi \in \operatorname{Irr}\left(\mathscr{M}_{*}\right)}^{\oplus} W(\pi)$, which is acting on the Hilbert space $\sum_{\pi \in \operatorname{Irr}\left(\mathscr{M}_{*}\right)}^{\oplus} L^{2}(\psi) \otimes \mathscr{H}_{\pi}$. Note that the Hilbert space $\sum_{\pi \in \operatorname{Irr}\left(\mathscr{M}_{*}\right)}^{\oplus} L^{2}(\psi) \otimes \mathscr{H}_{\pi}$ is canonically isomorphic to $L^{2}(\psi) \otimes \sum_{\pi \in \operatorname{Irr}\left(\mathscr{M}_{*}\right)}^{\oplus} \mathscr{H}_{\pi}$. The inverse $U$ of this isomorphism is characterized by

$$
U\left(\eta \otimes \sum_{\pi \in \operatorname{Irr}}^{\oplus} \xi_{\pi}\right)=\sum_{\pi \in \operatorname{Irr}}^{\oplus}\left(\eta \otimes \xi_{\pi}\right)
$$

By identifying $W_{\alpha}$ with $U^{*} W_{\alpha} U$, we shall often regard the unitary $W_{\alpha}$ as acting on $L^{2}(\psi) \otimes \sum_{\pi \in \operatorname{Irr}\left(\mathscr{M}_{*}\right)}^{\oplus} \mathscr{H}_{\pi}$. So, for example, we have

$$
\left(W_{\alpha}\left(\xi \otimes \sum_{\pi \in \operatorname{Irr}}^{\oplus} \xi_{\pi}\right) \mid \eta \otimes \sum_{\pi \in \operatorname{Irr}}^{\oplus} \eta_{\pi}\right)=\sum_{\pi \in \operatorname{Irr}}\left(W(\pi)\left(\xi \otimes \xi_{\pi}\right) \mid \eta \otimes \eta_{\pi}\right) .
$$

It is easily verified that, under the isomorphism $\operatorname{Ad} U^{*}$, the von Neumann algebra $\sum_{\pi \in \operatorname{Irr}\left(\mathscr{M}_{*}\right)}^{\oplus} \mathscr{A} \otimes \mathscr{L}\left(\mathscr{H}_{\pi}\right)$ is $*$-isomorphic to $\mathscr{A} \otimes \sum_{\pi \in \operatorname{Irr}\left(\mathscr{M}_{*}\right)}^{\oplus} \mathscr{L}\left(\mathscr{H}_{\pi}\right)$. Thus $W_{\alpha}$ belongs to $\mathscr{A} \otimes \sum_{\pi \in \operatorname{Irr}\left(\mathscr{M}_{*}\right)}^{\oplus} \mathscr{L}\left(\mathscr{H}_{\pi}\right)$. Let $\bar{\alpha}$ be the amplified action $\bar{\alpha}=\left(\mathrm{id}_{\mathscr{A}} \otimes \sigma\right) \circ(\alpha \otimes \mathrm{id})$ of $\mathrm{K}$ on $\mathscr{Q}:=\mathscr{A} \otimes \sum_{\pi \in \operatorname{Irr}\left(\mathscr{M}_{*}\right)} \mathscr{L}\left(\mathscr{H}_{\pi}\right)$, or appropriate von Neumann algebras. We also let $U_{\psi}$ be the canonical implementation of 
$\alpha$ on the standard Hilbert space $L^{2}(\psi)$ (see Part A of Appendix for details). With $\xi, \eta \in L^{2}(\psi)$ and $f, g \in L^{2}(\varphi)$, from (2.15), we have

$$
\begin{aligned}
\left(\left(\mathrm{id}_{2} \otimes \omega_{f, g}\right)(\right. & \left.\left.\bar{\alpha}\left(W_{\alpha}\right)\right)\left(\xi \otimes \sum_{\pi \in \operatorname{Irr}}^{\oplus} \xi_{\pi}\right) \mid \eta \otimes \sum_{\pi \in \operatorname{Irr}}{ }^{\oplus} \eta_{\pi}\right) \\
& =\left((\alpha \otimes \mathrm{id})\left(W_{\alpha}\right)\left(\xi \otimes f \otimes \sum_{\pi \in \operatorname{Irr}}^{\oplus} \xi_{\pi}\right) \mid \eta \otimes g \otimes \sum_{\pi \in \operatorname{Irr}}^{\oplus} \eta_{\pi}\right) \\
& =\left(\left(U_{\psi}\right)_{12}\left(W_{\alpha}\right)_{13}\left(U_{\psi}\right)_{12}^{*}\left(\xi \otimes f \otimes \sum_{\pi \in \operatorname{Irr}}^{\oplus} \xi_{\pi}\right) \mid \eta \otimes g \otimes \sum_{\pi \in \operatorname{Irr}}{ }^{\oplus} \eta_{\pi}\right) \\
& =\sum_{\pi \in \operatorname{Irr}}\left(\left(U_{\psi}\right)_{12} W(\pi)\left(U_{\psi}\right)_{12}^{*}\left(\xi \otimes f \otimes \xi_{\pi}\right) \mid \eta \otimes g \otimes \eta_{\pi}\right) \\
& =\sum_{\pi \in \operatorname{Irr}}\left(\bar{\alpha}(W(\pi))\left(\xi \otimes \xi_{\pi} \otimes f\right) \mid \eta \otimes \eta_{\pi} \otimes g\right) \\
& =\sum_{\pi \in \operatorname{Irr}}\left(W(\pi)_{12} V(\pi)_{23}\left(\xi \otimes \xi_{\pi} \otimes f\right) \mid \eta \otimes \eta_{\pi} \otimes g\right) \\
& =\sum_{\pi \in \operatorname{Irr}}<V(\pi)_{23}, \omega_{\xi \otimes \xi_{\pi}, W(\pi)^{*}\left(\eta \otimes \eta_{\pi}\right)} \otimes \omega_{f, g}> \\
& =\sum_{\pi \in \operatorname{Irr}}\left(W(\pi)\left(1 \otimes \pi\left(\omega_{f, g}\right)\right)\left(\xi \otimes \xi_{\pi}\right) \mid \eta \otimes \eta_{\pi}\right) \\
& =\left(W_{\alpha}\left(1 \otimes \sum_{\pi \in \operatorname{Irr}}^{\oplus} \pi\left(\omega_{f, g}\right)\right)\left(\xi \otimes \sum_{\pi \in \operatorname{Irr}}^{\oplus} \xi_{\pi}\right) \mid \eta \otimes \sum_{\pi \in \operatorname{Irr}}^{\oplus} \eta_{\pi}\right) .
\end{aligned}
$$

Thus we have shown that

$$
\left(\mathrm{id}_{\mathscr{Q}} \otimes \omega_{f, g}\right)\left(\bar{\alpha}\left(W_{\alpha}\right)\right)=W_{\alpha}\left(1 \otimes \sum_{\pi \in \operatorname{Irr}}^{\oplus} \pi\left(\omega_{f, g}\right)\right) .
$$

Since the direct sum representation $\sum_{\pi \in \operatorname{Irr}}^{\oplus} \pi$ is quasi-equivalent to the left regular (Fourier) represenation $\lambda$ of $\mathrm{K}$ by [ES1], there is a $*$-isomorphism $\Phi$ from $\sum_{\pi \in \operatorname{Irr}}^{\oplus} \mathscr{L}\left(\mathscr{H}_{\pi}\right)$ onto the dual Kac algebra $\hat{\mathscr{M}}$ such that $\Phi \circ\left(\sum_{\pi \in \operatorname{Irr}}^{\oplus} \pi\right)=\lambda$. Set $V_{\alpha}=\left(\operatorname{id}_{\mathscr{A}} \otimes \Phi\right)\left(W_{\alpha}\right)$, which lies in $\mathscr{A} \bar{\otimes} \hat{\mathscr{M}}$. Since

$$
\left(\mathrm{id}_{\mathscr{A}} \otimes \Phi \otimes \mathrm{id}_{\mathscr{M}}\right) \circ \bar{\alpha}\left(W_{\alpha}\right)=\bar{\alpha}\left(V_{\alpha}\right),
$$

it follows that we have

$$
\begin{aligned}
\left(\mathrm{id}_{\mathscr{A}} \otimes \Phi\right)\left(\mathrm{id}_{\mathscr{Q}} \otimes \omega_{f, g}\right)\left(\bar{\alpha}\left(W_{\alpha}\right)\right)= & \left(\mathrm{id}_{\mathscr{A}} \otimes \mathrm{id}_{\hat{M}} \otimes \omega_{f, g}\right) \\
& \circ\left(\operatorname{id}_{\mathscr{A}} \otimes \Phi \otimes \mathrm{id}_{\mathscr{M}}\right) \circ \bar{\alpha}\left(W_{\alpha}\right) \\
= & \left(\mathrm{id}_{\mathscr{A}} \otimes \mathrm{id}_{\hat{M}} \otimes \omega_{f, g}\right) \circ \bar{\alpha}\left(V_{\alpha}\right) .
\end{aligned}
$$


In the meantime, since the regular representation $\lambda$ has $\hat{W}=W(\hat{\mathrm{K}})$ as a generator, we have

$$
\left(\mathrm{id}_{\mathscr{A}} \otimes \Phi\right)\left(1 \otimes \sum_{\pi \in \operatorname{Irr}}^{\oplus} \pi\left(\omega_{f, g}\right)\right)=1 \otimes \lambda\left(\omega_{f, g}\right)=\left(\operatorname{id}_{\mathscr{A}} \otimes \operatorname{id}_{\hat{\mathscr{M}}} \otimes \omega_{f, g}\right)\left(\hat{W}_{23}\right) .
$$

Consequently, by applying $\operatorname{id}_{\mathscr{A}} \otimes \Phi$ to both sides of (2.16), we obtain

$$
\left(\mathrm{id}_{\mathscr{A}} \otimes \mathrm{id}_{\hat{\mathscr{M}}} \otimes \omega_{f, g}\right) \circ \bar{\alpha}\left(V_{\alpha}\right)=\left(\operatorname{id}_{\mathscr{A}} \otimes \mathrm{id}_{\hat{M}} \otimes \omega_{f, g}\right)\left(\left(V_{\alpha}\right)_{12} \hat{W}_{23}\right) .
$$

From this, it results that

$$
\bar{\alpha}\left(V_{\alpha}\right)=\left(V_{\alpha}\right)_{12} \hat{W}_{23} .
$$

Now we define an automorphism $\Theta$ of $\mathscr{A} \otimes \mathscr{L}\left(L^{2}(\varphi)\right)$ by $\Theta=\operatorname{Ad} V_{\alpha}$. We also consider the action $\tilde{\alpha}$ of $\mathrm{K}$ on $\mathscr{A} \otimes \mathscr{L}\left(L^{2}(\varphi)\right)$ introduced in Section 1. Then, from (2.17), we see that, for any $X \in \mathscr{A} \otimes \mathscr{L}\left(L^{2}(\varphi)\right)$, we have

$$
\begin{aligned}
\left(\Theta \otimes \operatorname{id}_{\mathscr{M}}\right) \circ \tilde{\alpha} \circ \Theta^{-1}(X) & =\left(V_{\alpha}\right)_{12} \tilde{\alpha}\left(V_{\alpha}^{*} X V_{\alpha}\right)\left(V_{\alpha}\right)_{12}^{*} \\
& =\left(V_{\alpha}\right)_{12} \hat{W}_{23} \bar{\alpha}\left(V_{\alpha}^{*} X V_{\alpha}\right) \hat{W}_{23}^{*}\left(V_{\alpha}\right)_{12}^{*}=\bar{\alpha}(X) .
\end{aligned}
$$

Thus we have proven that $\bar{\alpha}$ is conjugate to $\tilde{\alpha}$. This, together with Corollary B.2, implies that $\alpha$ is conjugate to $\tilde{\alpha}$. Since $\tilde{\alpha}$ is conjugate to the bidual action $\alpha$ of $\alpha$ by Proposition IV.5 of [ES2], we conclude that $\alpha$ is dual.

\section{On certain automorphisms in $\operatorname{Aut}\left(\mathscr{A} / \mathscr{A}^{\alpha}\right)$}

This section contains a study of certain special automorphisms in $\operatorname{Aut}\left(\mathscr{A} / \mathscr{A}^{\alpha}\right)$, with the emphasis on the relation with Galois correspondence of the inclusion $\mathscr{A}^{\alpha} \subseteq \mathscr{A}$. We show that such an automorphism gives rise to a unitary in the intrinsic group of the dual Kac algebra. It is also shown that the left co-ideal von Neumann subalgebra of $\mathrm{K}$ detemined by the fixed-point subfactor of the automorphism is explicitly described in terms of the unitary.

As in the preceding section, $\alpha$ is a minimal action of a compact Kac algebra $\mathrm{K}$ on a factor $\mathscr{A}$ with properly infinite fixed-point algebra. We denote by $\operatorname{Aut}\left(\mathscr{A} / \mathscr{A}^{\alpha}\right)$ the subgroup of all automorphisms of $\mathscr{A}$ leaving the fixedpoint algebra $\mathscr{A}^{\alpha}$ pointwise invariant. Let $\operatorname{Irr}=\operatorname{Irr}\left(\mathscr{M}_{*}\right)$ be as in the proof of Theorem 2.2. Next, for each $\left(\pi, \mathscr{H}_{\pi}\right) \in \operatorname{Irr}$ with generator $V(\pi)$, let $\left\{V(\pi)_{i, j}\right\}_{1 \leq i, j \leq d(\pi)}$ be as in the proof of Lemma 2.6, where $d(\pi)=\operatorname{dim} \mathscr{H}_{\pi}$. In view of Proposition 2.14, we may choose an $\alpha$-invariant Hilbert space $D_{\pi}$ in $\mathscr{A}$ and an ONB $\left\{w(\pi)_{i}\right\}_{1 \leq i \leq d(\pi)}$ for $D_{\pi}$ such that 
ON DOMINANCY OF MINIMAL ACTIONS OF COMPACT KAC ALGEBRAS... 309

$$
\alpha\left(w(\pi)_{j}\right)=\sum_{i=1}^{d(\pi)} w(\pi)_{i} \otimes V(\pi)_{i, j} . \quad(1 \leq j \leq d(\pi))
$$

Suppose now that $\theta \in \operatorname{Aut}\left(\mathscr{A} / \mathscr{A}^{\alpha}\right)$, which leaves each of the $\alpha$-invariant Hilbert spaces in $\mathscr{A}$ globally invariant, is given. Then set

$$
V(\theta(\pi))_{i, j}=\sum_{k=1}^{d(\pi)}\left(w(\pi)_{k} \mid \theta\left(w(\pi)_{i}\right)\right) V(\pi)_{k, j} \quad(1 \leq i, j \leq d(\pi), \pi \in \operatorname{Irr})
$$

For each $\pi \in \operatorname{Irr}$, consider the finite-dimensional Hilbert space $\mathfrak{M}_{\pi}=\operatorname{span}\left\{\Lambda_{\varphi}\left(V(\pi)_{i, j}\right): 1 \leq i, j \leq d(\pi)\right\}$ of $L^{2}(\varphi)$. The equation

$$
u(\theta) \Lambda_{\varphi}\left(V(\pi)_{i, j}\right)=\Lambda_{\varphi}\left(V(\theta(\pi))_{i, j}\right)
$$

defines a linear transformation $u(\theta)$ on $\mathfrak{M}_{\pi}$. In fact, since $\left\{\sqrt{d(\pi)} \Lambda_{\varphi}\left(V(\pi)_{i, j}\right)\right\}$ is an ONB for $\mathfrak{M}_{\pi}$, it easily follows that $u(\theta)$ is a unitary. Since $L^{2}(\varphi)=\sum_{\pi \in \operatorname{Irr}}^{\oplus} \mathfrak{M}_{\pi}, u(\theta)$ naturally extends to a unitary on $L^{2}(\varphi)$, which we denote by $u(\theta)$ again.

Lemma 3.3. The unitary $u(\theta)$ belongs to the dual Kac algebra $\hat{M}$.

Proof. Note first that the commutant $\hat{M}^{\prime}$ is generated by the right regular representation $\lambda^{\prime}$ of $\mathscr{M}_{*}$ defined by $\lambda^{\prime}(\omega)=\hat{J} \lambda(\omega)^{*} \hat{J}$. Here $\hat{J}$ stands for the modular conjugation of the dual Haar measure $\hat{\varphi}$ which is given by $\hat{J} \Lambda_{\varphi}(x)=\Lambda_{\varphi}\left(\kappa\left(x^{*}\right)\right)$. From Theorem 2.5.3 of [ES1], it is easily verified that

$$
\lambda^{\prime}(\omega) \Lambda_{\varphi}(x)=\Lambda_{\varphi}\left(\left(\operatorname{id}_{\mathscr{M}} \otimes \omega \circ \kappa\right) \circ \Gamma(x)\right) \quad(x \in \mathscr{M}) .
$$

From this, together with the fact that $\Gamma\left(V(\pi)_{i, j}\right)=\sum_{k=1}^{d(\pi)} V(\pi)_{i, k} \otimes V(\pi)_{k, j}$, it follows that, for any $\omega \in \mathscr{M}_{*}$, we have

$$
\begin{aligned}
u(\theta) \lambda^{\prime}(\omega) \Lambda_{\varphi}\left(V(\pi)_{i, j}\right) & =u(\theta) \Lambda_{\varphi}\left(\left(\left(\operatorname{id}_{\mathscr{M}} \otimes \omega \circ \kappa\right) \circ \Gamma\left(V(\pi)_{i, j}\right)\right)\right. \\
& =\sum_{k=1}^{d(\pi)} \omega \circ \kappa\left(V(\pi)_{k, j}\right) u(\theta) \Lambda_{\varphi}\left(V(\pi)_{i, k}\right) \\
& =\sum_{k, \ell=1}^{d(\pi)}\left(w(\pi)_{\ell} \mid \theta\left(w(\pi)_{i}\right)\right) \omega \circ \kappa\left(V(\pi)_{k, j}\right) \Lambda_{\varphi}\left(V(\pi)_{\ell, k}\right) \\
& =\sum_{\ell=1}^{d(\pi)}\left(w(\pi)_{\ell} \mid \theta\left(w(\pi)_{i}\right)\right) \Lambda_{\varphi}\left(\left(\operatorname{id}_{\mathscr{M}} \otimes \omega \circ \kappa\right) \circ \Gamma\left(V(\pi)_{\ell, j}\right)\right) \\
& =\sum_{\ell=1}^{d(\pi)}\left(w(\pi)_{\ell} \mid \theta\left(w(\pi)_{i}\right)\right) \lambda^{\prime}(\omega) \Lambda_{\varphi}\left(V(\pi)_{\ell, j}\right) \\
& =\lambda^{\prime}(\omega) u(\theta) \Lambda_{\varphi}\left(V(\pi)_{i, j}\right) .
\end{aligned}
$$


This completes the proof.

LEMMA 3.4. We have

$$
V\left(\theta\left(\pi_{1}\right)\right)_{i, j} u(\theta) \Lambda_{\varphi}\left(V\left(\pi_{2}\right)_{k, \ell}\right)=u(\theta) V\left(\pi_{1}\right)_{i, j} \Lambda_{\varphi}\left(V\left(\pi_{2}\right)_{k, \ell}\right)
$$

for any $\pi_{1}, \pi_{2} \in \operatorname{Irr}\left(\mathscr{M}_{*}\right)$.

Proof. Let $\pi_{1}, \pi_{2}$ be in $\operatorname{Irr}\left(\mathscr{M}_{*}\right)$. We write $D_{1}, D_{2}$ for $D_{\pi_{1}}, D_{\pi_{2}}$, respectively for simplicity. It is easy to see that the representation $\pi_{D_{1} D_{2}}$ associated with the $\alpha$-invariant Hilbert space $D_{1} D_{2}$ is unitarily equivalent to the Kronecker product $\pi_{1} \times \pi_{2}$ (see Theorem 1.4.3 of [ES1]). Moreover, we have

$$
\begin{aligned}
& \left(\pi_{D_{1} D_{2}}(\omega) w\left(\pi_{1}\right)_{j} w\left(\pi_{2}\right)_{k} \mid w\left(\pi_{1}\right)_{m} w\left(\pi_{2}\right)_{n}\right) \\
& =\omega\left(V\left(\pi_{1}\right)_{m, j} V\left(\pi_{2}\right)_{n, k}\right) \quad\left(\omega \in \mathscr{M}_{*}\right) .
\end{aligned}
$$

By using 3.5 and the Fourier expansion with repect to the ONB $\left\{\sqrt{d(\pi)} \Lambda_{\varphi}\left(V(\pi)_{i, j}\right): 1 \leq i, j \leq d(\pi), \pi \in \operatorname{Irr}\right\}$, we have

$$
\begin{aligned}
& V\left(\theta\left(\pi_{1}\right)\right)_{i, j} u(\theta) \Lambda_{\varphi}\left(V\left(\pi_{2}\right)_{k, \ell}\right) \sum_{m=1}^{d\left(\pi_{1}\right)} \sum_{n=1}^{d\left(\pi_{2}\right)}\left(w\left(\pi_{1}\right)_{m} w\left(\pi_{2}\right)_{n} \mid \theta\left(w\left(\pi_{1}\right)_{i} w\left(\pi_{2}\right)_{k}\right)\right) V\left(\pi_{1}\right)_{m, j} \Lambda_{\varphi}\left(V\left(\pi_{2}\right)_{n, \ell}\right) \\
&= \sum_{m=1}^{d\left(\pi_{1}\right)} \sum_{n=1}^{d\left(\pi_{2}\right)} \sum_{\pi \in \operatorname{Irr}} d(\pi) \sum_{p, q=1}^{d(\pi)}\left(w\left(\pi_{1}\right)_{m} w\left(\pi_{2}\right)_{n} \mid \theta\left(w\left(\pi_{1}\right)_{i} w\left(\pi_{2}\right)_{k}\right)\right) \\
&= \sum_{m=1}^{d\left(\pi_{1}\right)} \sum_{n=1}^{d\left(\pi_{2}\right)} \sum_{\pi \in \operatorname{Irr}} d(\pi) \sum_{p, q=1}^{d(\pi)}\left(w\left(\pi_{1}\right)_{m, j} \Lambda_{\varphi}\left(V\left(\pi_{2}\right)_{n, \ell}\right) \mid \Lambda_{\varphi}\left(V(\pi)_{p, q}\right) \Lambda_{\varphi}\left(V(\pi)_{p, q}\right)\right. \\
& \times\left(\pi_{D_{1} D_{2}}\left(\varphi V(\pi)_{p, q}^{*}\right) w\left(\pi_{1}\right)_{j} w\left(\pi_{2}\right)_{\ell} \mid w\left(\pi_{1}\right)_{m} w\left(\pi_{2}\right)_{n}\right) \Lambda_{\varphi}\left(V(\pi)_{p, q}\right) \\
&=\sum_{\substack{\pi \in \operatorname{Irr} ; \\
\pi<\pi_{1} \times \pi_{2}}} d(\pi) \sum_{p, q=1}^{d(\pi)}\left(\pi_{D_{1} D_{2}}\left(\varphi V(\pi)_{p, q}^{*}\right) w\left(\pi_{1}\right)_{j} w\left(\pi_{2}\right)_{\ell} \mid\right. \\
&\left.\times \theta\left(w\left(\pi_{1}\right)_{i} w\left(\pi_{2}\right)_{k}\right)\right) \Lambda_{\varphi}\left(V(\pi)_{p, q}\right) .
\end{aligned}
$$

In the meantime, similar calculations show that $u(\theta) V\left(\pi_{1}\right)_{i, j} \Lambda_{\varphi}\left(V\left(\pi_{2}\right)_{k, \ell}\right)$ also equals the vector ocurring last in the above computation. The details are left to readers. 
ON DOMINANCY OF MINIMAL ACTIONS OF COMPACT KAC ALGEBRAS... 311

Proposition 3.6. The unitary $u(\theta)$ satisfies

$$
W(u(\theta) \otimes u(\theta))=(u(\theta) \otimes 1) W .
$$

In particular, $u(\theta)$ belongs to the intrinsic group $G(\hat{\mathrm{K}})$ of $\hat{\mathrm{K}}$.

Proof. Let $\pi_{1}, \pi_{2}$ be in $\operatorname{Irr}\left(\mathscr{M}_{*}\right)$. Then, by Lemma 3.4, we have

$$
\begin{aligned}
W(u(\theta) \otimes u(\theta))\left(\Lambda_{\varphi}\left(V\left(\pi_{1}\right)_{i, j}\right) \otimes \Lambda_{\varphi}\left(V\left(\pi_{2}\right)_{k, \ell}\right)\right) \\
=\sum_{m=1}^{d\left(\pi_{1}\right)} \sum_{n=1}^{d\left(\pi_{2}\right)}\left(w\left(\pi_{1}\right)_{m} \mid \theta\left(w\left(\pi_{1}\right)_{i}\right)\right)\left(w\left(\pi_{2}\right)_{n} \mid \theta\left(w\left(\pi_{2}\right)_{k}\right)\right) \\
\quad \times W\left(\Lambda_{\varphi}\left(V\left(\pi_{1}\right)_{m, j}\right) \otimes \Lambda_{\varphi}\left(V\left(\pi_{2}\right)_{n, \ell}\right)\right) \\
=\sum_{m=1}^{d\left(\pi_{1}\right)} \sum_{n=1}^{d\left(\pi_{2}\right)} \Lambda_{\varphi \otimes \varphi}\left(\Gamma\left(V\left(\pi_{2}\right)_{n, \ell}\right)\left(V\left(\pi_{1}\right)_{m, j} \otimes 1\right)\right) \\
=\sum_{m=1}^{d\left(\pi_{1}\right)} \sum_{n=1}^{d\left(\pi_{2}\right)} \sum_{p=1}^{d\left(\pi_{2}\right)} \Lambda_{\varphi}\left(V\left(\pi_{2}\right)_{n, p} V\left(\pi_{1}\right)_{m, j}\right) \otimes \Lambda_{\varphi}\left(V\left(\pi_{2}\right)_{p, \ell}\right) \\
=\sum_{p=1}^{d\left(\pi_{2}\right)} u(\theta) V\left(\pi_{2}\right)_{k, p} \Lambda_{\varphi}\left(V\left(\pi_{1}\right)_{i, j}\right) \otimes \Lambda_{\varphi}\left(V\left(\pi_{2}\right)_{p, \ell}\right) \\
=(u(\theta) \otimes 1) \Lambda_{\varphi \otimes \varphi}\left(\Gamma\left(V\left(\pi_{2}\right)_{k, \ell}\right)\left(V\left(\pi_{1}\right)_{i, j} \otimes 1\right)\right) \\
=(u(\theta) \otimes 1) W\left(\Lambda_{\varphi}\left(V\left(\pi_{1}\right)_{i, j}\right) \otimes \Lambda_{\varphi}\left(V\left(\pi_{2}\right)_{k, \ell}\right)\right) .
\end{aligned}
$$

Since the set $\left\{\sqrt{d(\pi)} \Lambda_{\varphi}\left(V(\pi)_{i, j}\right): 1 \leq i, j \leq d(\pi), \pi \in \operatorname{Irr}\right\}$ is total in $L^{2}(\varphi)$, the first assertion follows.

For the second assertion, it suffices to note that $W(\hat{\mathrm{K}})=\Sigma W^{*} \Sigma$.

In order to relate our results to Galois correspondence established in [ILP], we modify the unitary $u(\theta)$ a little so that it may fit into their theory. Set $v(\theta)=\hat{J} u(\theta) \hat{J}$, which belongs to the Kac algebra $\hat{K}^{\prime}$. We emphasize that, in [ILP], it is $\hat{K}^{\prime}$ that is considered to be the Kac algebra dual to K. By (3.1) and (3.2), we have

$$
v(\theta) \Lambda_{\varphi}\left(V(\pi)_{i, j}\right)=\Lambda_{\varphi}\left(V(\theta(\pi))_{i, j}^{\prime}\right),
$$

where $V(\theta(\pi))_{i, j}^{\prime}=\sum_{k=1}^{d(\pi)}\left(\theta\left(w(\pi)_{j}\right) \mid w(\pi)_{k}\right) V(\pi)_{i, k}$. By Proposition 3.6, $v(\theta)$ belongs to the intrinsic group $G\left(\hat{K}^{\prime}\right)$. It follows from Theorem 2.3 of [DeC] that $\beta_{\theta}=\operatorname{Ad} v(\theta)$ defines an automorphism of $\mathbf{K}^{\sigma}=\left(\hat{\mathrm{K}}^{\prime}\right)^{\wedge}$ satisfying $\left(\beta_{\theta} \otimes \operatorname{id}_{\mathscr{M}}\right) \circ \Gamma^{\sigma}=\Gamma^{\sigma} \circ \beta_{\theta}$, whose canonical implementation on $L^{2}(\varphi)$ is precisely $v(\theta)$. From Lemma 3.4, we find that $u(\theta) V(\pi)_{i, j} u(\theta)^{*}=V(\theta(\pi))_{i, j}$. By applying Ad $\hat{J}$ to both sides of this identity, we obtain $v(\theta) V(\pi)_{i, j} v(\theta)^{*}=$ $V(\theta(\pi))_{i, j}^{\prime}$. Thus the automorphism $\beta_{\theta}$ enjoys the following properties: 


$$
\begin{aligned}
& \left(\operatorname{id}_{\mathscr{M}} \otimes \beta_{\theta}\right) \circ \Gamma=\Gamma \circ \beta_{\theta}, \\
& \beta_{\theta}\left(V(\pi)_{i, j}\right)=V(\theta(\pi))_{i, j}^{\prime} \quad(1 \leq i, j \leq d(\pi), \pi \in \operatorname{Irr}) .
\end{aligned}
$$

Lemma 3.9. Let $\pi$ be in $\operatorname{Irr}\left(\mathscr{M}_{*}\right)$. Then the $\pi$-spectral subspace $\mathscr{A}_{\pi}$ introduced in Part A of Appendix has the form $\mathscr{A}_{\pi}=\mathscr{A}^{\alpha} D_{\pi}$.

Proof. Let $D$ be an $\alpha$-invariant subspace of $\mathscr{A}$ such that $\pi_{D}$ is equivalent to $\pi$ in the sense of Lemma 2.6. Then, as in the proof of Lemma 2.6, we may choose a basis $\left\{v_{i}\right\}$ of $D$ such that

$$
\alpha\left(v_{j}\right)=\sum_{i=1}^{d(\pi)} v_{i} \otimes V(\pi)_{i, j}
$$

Then we have

$$
\alpha\left(w(\pi)_{j} v_{j}^{*}\right)=\sum_{i, k=1}^{d(\pi)} w(\pi)_{k} v_{i}^{*} \otimes V(\pi)_{k, j} V(\pi)_{i, j}^{*} .
$$

Since $E_{\alpha}=\left(\operatorname{id}_{\mathscr{A}} \otimes \varphi\right) \circ \alpha$,

$$
E_{\alpha}\left(w(\pi)_{j} v_{j}^{*}\right)=\sum_{i, k=1}^{d(\pi)} \varphi\left(V(\pi)_{k, j} V(\pi)_{i, j}^{*}\right) w(\pi)_{k} v_{i}^{*}=\frac{1}{d(\pi)} \sum_{i=1}^{d(\pi)} w(\pi)_{i} v_{i}^{*}
$$

Thus $z=\sum_{i=1}^{d(\pi)} w(\pi)_{i} v_{i}^{*}$ belongs to $\mathscr{A}^{\alpha}$, whence $v_{i}=z^{*} w(\pi)_{i} \in \mathscr{A}^{\alpha} D_{\pi}$. In view of Proposition A.1, we obtain $\mathscr{A}_{\pi} \subseteq \mathscr{A}^{\alpha} D_{\pi}$. The reverse inclusion is clear.

Proposition 3.10. We have

$$
\left(\mathrm{id}_{\mathscr{A}} \otimes \beta_{\theta}\right) \circ \alpha=\alpha \circ \theta .
$$

Proof. First, a straightforward calculation yields

$$
\sum_{i=1}^{d(\pi)} w(\pi)_{i} \otimes V(\theta(\pi))_{i, j}^{\prime}=\alpha\left(\theta\left(w(\pi)_{j}\right)\right) .
$$

With $z \in \mathscr{A}^{\alpha}$, by (3.8) and (3.11), we obtain

$$
\begin{aligned}
\left(\mathrm{id}_{\mathscr{A}} \otimes \beta_{\theta}\right) \circ \alpha\left(z w(\pi)_{j}\right) & =\sum_{i=1}^{d(\pi)}\left(\mathrm{id}_{\mathscr{A}} \otimes \beta_{\theta}\right)\left(z w(\pi)_{i} \otimes V(\pi)_{i, j}\right) \\
& =\sum_{i=1}^{d(\pi)} z w(\pi)_{i} \otimes V(\theta(\pi))_{i, j}^{\prime} \\
& =(z \otimes 1) \alpha\left(\theta\left(w(\pi)_{j}\right)\right)=\alpha \circ \theta\left(z w(\pi)_{j}\right) .
\end{aligned}
$$

From this and Lemma 3.9, it follows that the assertion is true for any ele- 
ment of the $\pi$-spectral subspace $\mathscr{A}_{\pi}$. Since the subspace generated by all the $\pi$-spectral subspaces is $\sigma$-weakly dense in $\mathscr{A}$, we obtain the asserted identity.

Now let us consider the fixed-point algebra $\mathscr{A}^{\theta}$ of the automrphism $\theta$. Since $\theta \in \operatorname{Aut}\left(\mathscr{A} / \mathscr{A}^{\alpha}\right)$, the von Neumann algebra $\mathscr{A}^{\theta}$ is an intermediate subfactor of the inclusion $\mathscr{A}^{\alpha} \subseteq \mathscr{A}$. According to [ILP], there exists a unique left co-ideal von Neumann subalgebra $\mathscr{N}(\theta)$ of $\mathscr{M}$ such that

$$
\mathscr{A}^{\theta}=\{x \in \mathscr{A}: \alpha(x) \in \mathscr{A} \otimes \mathscr{N}(\theta)\} .
$$

The following theorem illustrates how $\mathscr{N}(\theta)$ can be described in terms of the unitary $v(\theta)$.

TheOrem 3.12. The left co-ideal von Neumann subalgebra $\mathscr{N}(\theta)$ of $\mathscr{M}$ determined by the intermediate subfactor $\mathscr{A}^{\theta}$ is the fixed-point algebra $\mathscr{M}^{\beta_{\theta}}$ of the associated automorphism $\beta_{\theta}$ of $\mathscr{M}$. Thus we have

$$
\mathscr{N}(\theta)=\{a \in \mathscr{M}: a v(\theta)=v(\theta) a\} .
$$

Proof. By (3.8), the fixed-point algebra $\mathscr{M}^{\beta_{\theta}}$ is a left co-ideal von Neumann subalgebra of $\mathscr{M}$. Hence, by Theorem 4.4 of [ILP], $\mathscr{M}^{\beta_{\theta}}$ determines a unique intermediate subfactor $\mathscr{B}$ of the inclusion $\mathscr{A}^{\alpha} \subseteq \mathscr{A}$. So we have

$$
\mathscr{B}=\left\{x \in \mathscr{A}: \alpha(x) \in \mathscr{A} \otimes \mathscr{M}^{\beta_{\theta}}\right\} .
$$

Now let $x$ be in $\mathscr{A}^{\theta}$. It then follows from Proposition 3.10 that $\alpha(x)$ belongs to the fixed-point algebra $(\mathscr{A} \bar{\otimes} \mathscr{M})^{\text {id }_{\mathscr{A}} \otimes \beta_{\theta}}=\mathscr{A} \otimes \mathscr{M}^{\beta_{\theta}}$. Thus $x \in \mathscr{B}$.

Conversely, suppose that $x \in \mathscr{B}$. Then, by Proposition 3.10, we have

$$
\alpha \circ \theta(x)=\left(\operatorname{id}_{\mathscr{A}} \otimes \beta_{\theta}\right) \circ \alpha(x)=\alpha(x) .
$$

Hence $\theta(x)=x$, i.e., $x \in \mathscr{A}^{\theta}$.

Consequently, $\mathscr{A}^{\theta}=\mathscr{B}$. Therefore, the corresponding left co-ideal von Neumann subalgebras $\mathscr{N}(\theta)$ and $\mathscr{M}^{\beta_{\theta}}$ must coincide with each other.

\section{Appendix}

This appendix is concerned with some general results on actions of Kac algebras on von Neumann algebras. We believe that most of our results are well-known to experts. But the author was unable to locate a literature that actually contains them. So we provide their proofs for readers' convenience.

We shall keep the notation in Section 1. Throughout this appendix, we fix an action $\alpha$ of $\mathrm{K}$ on a von Neumann algebra $\mathscr{P}$.

Part A. Spectral subspaces. In this subsection, we briefly study the spectral subspaces associated with a compact Kac algebra action. So let us assume 
for the time being that $\mathrm{K}$ is compact. We use the normalized Haar measure $\varphi$.

For each irreducible representation $\left(\pi, \mathscr{H}_{\pi}\right)$ of $\mathscr{M}_{*}$ with generator $V(\pi)$, let $\chi_{\pi}$ denote the normalized character of $\pi$ (c.f. [KP], [W]), i.e., $\chi_{\pi}=d(\pi)\left(\operatorname{Tr}_{\mathscr{H}_{\pi}} \otimes \operatorname{id}_{\mathscr{M}}\right)(V(\pi)) \in \mathscr{M}$, where $d(\pi)=\operatorname{dim} \mathscr{H}_{\pi}$, and $\operatorname{Tr}_{\mathscr{H}}$ in general stands for the usual trace on the finite-dimensional algebra $\mathscr{L}(\mathscr{H})$. Then we define a normal linear map $E_{\pi}: \mathscr{P} \rightarrow \mathscr{P}$ by

$$
E_{\pi}=\left(\operatorname{id}_{\mathscr{P}} \otimes \chi_{\pi}^{*} \varphi\right) \circ \alpha .
$$

Since $\chi_{\pi}^{*} \varphi$ belongs to the center of the Banach algebra $\mathscr{M}_{*}$, we have $\left(\operatorname{id}_{\mathscr{M}} \otimes \chi_{\pi}^{*} \varphi\right) \circ \Gamma=\left(\chi_{\pi}^{*} \varphi \otimes \operatorname{id}_{\mathscr{M}}\right) \circ \Gamma$. From this, it results that, for any $x \in \mathscr{P}$,

$$
\begin{aligned}
\alpha\left(E_{\pi}(x)\right) & =\left(\alpha \otimes \chi_{\pi}^{*} \varphi\right) \circ \alpha(x)=\left(\operatorname{id}_{\mathscr{P}} \otimes \operatorname{id}_{\mathscr{M}} \otimes \chi_{\pi}^{*} \varphi\right) \circ\left(\alpha \otimes \operatorname{id}_{\mathscr{M}}\right) \circ \alpha(x) \\
& =\left(\operatorname{id}_{\mathscr{P}} \otimes \operatorname{id}_{\mathscr{M}} \otimes \chi_{\pi}^{*} \varphi\right) \circ\left(\operatorname{id}_{\mathscr{P}} \otimes \Gamma\right) \circ \alpha(x) \\
& =\left(\operatorname{id}_{\mathscr{P}} \otimes \chi_{\pi}^{*} \varphi \otimes \operatorname{id}_{\mathscr{M}}\right) \circ\left(\operatorname{id}_{\mathscr{P}} \otimes \Gamma\right) \circ \alpha(x) \\
& =\left(\operatorname{id}_{\mathscr{P}} \otimes \chi_{\pi}^{*} \varphi \otimes \operatorname{id}_{\mathscr{M}}\right) \circ\left(\alpha \otimes \operatorname{id}_{\mathscr{M}}\right) \circ \alpha(x)=\left(E_{\pi} \otimes \operatorname{id}_{\mathscr{M}}\right) \circ \alpha(x) .
\end{aligned}
$$

This shows that

$$
\alpha \circ E_{\pi}=\left(E_{\pi} \otimes \operatorname{id}_{\mathscr{M}}\right) \circ \alpha .
$$

Moreover, by using the fact that $\chi_{\pi}^{*} \varphi$ is a projection: $\chi_{\pi}^{*} \varphi * \chi_{\pi}^{*} \varphi=\chi_{\pi}^{*} \varphi$, we can prove that $E_{\pi} \circ E_{\pi}=E_{\pi}$. From these properties of $E_{\pi}$, it follows that the range $\mathscr{P}_{\pi}=E_{\pi}(\mathscr{P})$ is a $\sigma$-weakly closed subspace of $\mathscr{P}$. We call $\mathscr{P}_{\pi}$ the $\pi$ spectral subspace of $\mathscr{P}$ with respect to $\alpha$.

Let $D$ be an $\alpha$-invariant subspace of $\mathscr{P}$ such that $\pi_{D}$ is equivalent to $\pi$ (see Section 2). Then, as in the proof Lemma 2.6, we may choose a basis $\left\{v_{i}\right\}_{i=1}^{d=d(\pi)}$ of $D$ so that we have

$$
\alpha\left(v_{j}\right)=\sum_{i=1}^{d} v_{i} \otimes V(\pi)_{i, j},
$$

where $V(\pi)_{i, j}$ 's of course denote the matrix elements of $V(\pi)$ with respect to the ONB of $\mathscr{H}_{\pi}$ determined by $\left\{v_{i}\right\}$. From this, we have

$$
E_{\pi}\left(v_{j}\right)=\sum_{i=1}^{d} \varphi\left(\chi_{\pi}^{*} V(\pi)_{i, j}\right) v_{i}=d(\pi) \sum_{i, k=1}^{d} \varphi\left(V(\pi)_{k, k}^{*} V(\pi)_{i, j}\right)=\sum_{i, k=1}^{d} \delta_{i, k} \delta_{j, k} v_{i}=v_{j} .
$$

Here we used the fact that $\left\{\sqrt{d(\pi)} V(\pi)_{i, j}\right\}_{1 \leq i, j \leq d}$ forms an orthonormal system in $L^{2}(\varphi)$ (see [ES1]). Hence $D$ is contained in $\mathscr{P}_{\pi}$. Therefore, the $\pi$ spectral subspace $\mathscr{P}_{\pi}$ contains the subspace $\mathscr{D}_{\pi}$ of $\mathscr{P}$ generated by the family of $\alpha$-invariant subspaces $D$ with the property that $\pi_{D}$ is equivalent to $\pi$. 
ON DOMINANCY OF MINIMAL ACTIONS OF COMPACT KAC ALGEBRAS... 315

Conversely, let $x \in \mathscr{P}_{\pi}$, and consider the subspace $D_{x}$ defined by

$$
D_{x}=\left\{\left(\operatorname{id}_{\mathscr{P}} \otimes \omega\right) \circ \alpha(x): \omega \in \mathscr{M}_{*}\right\} .
$$

Since $E_{\pi}(x)=x, x$ lies in $D_{x}$. We assert that $D_{x}$ is finite-dimensional. For this, we need to introduce the canonical implementation of $\alpha$. Let $\omega_{0}$ be a faithful normal state on $\mathscr{P}^{\alpha}$, and $E_{\alpha}$ the faithful normal conditional expectation from $\mathscr{P}$ onto $\mathscr{P}^{\alpha}$ given by $E_{\alpha}=\left(\operatorname{id}_{\mathscr{P}} \otimes \varphi\right) \circ \alpha\left(E_{\alpha}\right.$ is nothing but $E_{\iota}$ in the above notation, where $\iota$ is the trivial representation of $\mathscr{M}_{*}$ ). Set $\psi=\omega_{0} \circ E_{\alpha}$, which is a faithful normal state on $\mathscr{P}$. As in Section 2, we regard $\mathscr{P}$ as represented on the Hilbert space $L^{2}(\psi)$ obtained from $\psi$ by the GNS construction. Let $\Lambda_{\psi}$ be the canonical injection $\Lambda_{\psi}: \mathscr{P} \rightarrow L^{2}(\psi)$. Then, by Section III of [ES2], the operator $U_{\psi}$ on $L^{2}(\psi) \otimes L^{2}(\varphi)$ defined by

$$
U_{\psi}\left(\Lambda_{\psi}(y) \otimes \Lambda_{\varphi}(a)\right)=\Lambda_{\psi \otimes \varphi}(\alpha(y)(1 \otimes a)) \quad(y \in \mathscr{P}, a \in \mathscr{M})
$$

is a unitary in $\mathscr{L}\left(L^{2}(\psi)\right) \bar{\otimes} \mathscr{M}$. Among others, it satisfies (i) $\left(U_{\psi}\right)_{12}\left(U_{\psi}\right)_{13}=$ $\left(\operatorname{id}_{\mathscr{L}\left(L^{2}(\psi)\right)} \otimes \Gamma\right)\left(U_{\psi}\right) ; \quad$ (ii) $\quad\left(\operatorname{id}_{\mathscr{L}\left(L^{2}(\psi)\right)} \otimes \omega \circ \kappa\right)\left(U_{\psi}\right)=\left(\operatorname{id}_{\mathscr{L}\left(L^{2}(\psi)\right)} \otimes \omega\right)\left(U_{\psi}^{*}\right)$ $\left(\omega \in \mathscr{M}_{*}\right)$; (iii) $\alpha(y)=U_{\psi}(y \otimes 1) U_{\psi}^{*}(y \in \mathscr{P})$. The unitary $U_{\psi}$ is called the canonical implementation on $L^{2}(\psi)$. From (i), (ii) and 1.5.2 of [ES1],

$$
\mu_{\alpha}(\omega)=(\mathrm{id} \otimes \omega)\left(U_{\psi}\right) \quad\left(\omega \in \mathscr{M}_{*}\right)
$$

defines a nondegenerate representation $\mu_{\alpha}$ of $\mathscr{M}_{*}$ on $L^{2}(\psi)$. With the notation just introduced, for any $a \in \mathscr{M}$ and $y \in \mathscr{P}$, we have

$$
\begin{aligned}
\left(\Lambda_{\psi}\left(\left(\operatorname{id}_{\mathscr{P}} \otimes \varphi_{a}\right)(\alpha(x))\right) \mid \Lambda_{\psi}(y)\right) & =(\psi \otimes \varphi)\left(\left(y^{*} \otimes a\right) \alpha(x)\right) \\
& =\left(\Lambda_{\psi \otimes \varphi}(\alpha(x)) \mid \Lambda_{\psi}(y) \otimes \Lambda_{\varphi}\left(a^{*}\right)\right) \\
& =\left(U_{\psi}\left(\Lambda_{\psi}(x) \otimes \Lambda_{\varphi}(1) \mid \Lambda_{\psi}(y) \otimes \Lambda_{\varphi}\left(a^{*}\right)\right)\right. \\
& =\left(\mu_{\alpha}\left(\varphi_{a}\right) \Lambda_{\psi}(x) \mid \Lambda_{\psi}(y)\right),
\end{aligned}
$$

where $\varphi_{a}$ is defined by $\varphi_{a}(b)=\varphi(a b)$. Thus we have shown that $\Lambda_{\psi}\left(\left(\operatorname{id}_{\mathscr{P}} \otimes \varphi_{a}\right)(\alpha(x))\right)=\mu_{\alpha}\left(\varphi_{a}\right) \Lambda_{\psi}(x)$. Since $\varphi_{a}$ 's form a dense subspace of $\mathscr{M}_{*}$, it follows that

$$
\begin{aligned}
& \Lambda_{\psi}\left(\left(\operatorname{id}_{\mathscr{P}} \otimes \omega\right)(\alpha(x))\right)=\mu_{\alpha}(\omega) \Lambda_{\psi}(x), \\
& \Lambda_{\psi}\left(D_{x}\right)=\left\{\mu_{\alpha}(\omega) \Lambda_{\psi}(x): \omega \in \mathscr{M}_{*}\right\} .
\end{aligned}
$$

Since $\Lambda_{\psi}(x)=\Lambda_{\psi}\left(E_{\pi}(x)\right)=\Lambda_{\psi}\left(\left(\mathrm{id} \otimes \chi_{\pi}^{*} \varphi\right)(\alpha(x))\right)=\mu_{\alpha}\left(\chi_{\pi}^{*} \varphi\right) \Lambda_{\psi}(x), \Lambda_{\psi}\left(D_{x}\right)$ is essentially the subspace $\left\{\mu_{\alpha}\left(\omega * \chi_{\pi}^{*} \varphi\right) \Lambda_{\psi}(x): \omega \in \mathscr{M}_{*}\right\}$. By using the Fourier expansion, we find that $\omega * \chi_{\pi}^{*} \varphi=d(\pi) \sum_{i, j=1}^{d(\pi)} \omega\left(V(\pi)_{i, j}\right)\left(V(\bar{\pi})_{i, j} \varphi\right)$ for any $\omega \in \mathscr{M}_{*}$. From this, it follows that $\Lambda_{\psi}\left(D_{x}\right)$ is generated by $\left\{\mu_{\alpha}\left(V(\bar{\pi})_{i, j} \varphi\right) \Lambda_{\psi}(x)\right\}_{1 \leq i, j \leq d(\pi)}$. In particular, $D_{x}$ itself is finite-dimensional.

Finally, we claim that $x \in \mathscr{D}_{\pi}$. By A. $1, \Lambda_{\psi}\left(D_{x}\right)$ is a $\mu_{\alpha}$-invariant subspace, 
so that $\left.\mu_{\alpha}\right|_{\Lambda_{\psi}\left(D_{x}\right)}$ can be decomposed into irreducible representations as follows: $\Lambda_{\psi}\left(D_{x}\right) \stackrel{=}{=} \sum_{\iota \in I}^{\oplus} \Lambda_{\psi}\left(D_{\iota}\right),\left.\mu_{\alpha}\right|_{\Lambda_{\psi}\left(D_{\iota}\right.} \cong \pi$. Consequently, $D_{x}$ is an algebraic direct sum: $D_{x}=\sum_{\iota \in I}^{\oplus} D_{\iota}$. In particular, $x$ can be written in the form: $x=\sum_{\iota \in I} x_{\iota}\left(x_{\iota} \in D_{\iota}\right)$. Hence, in order to show that $x \in \mathscr{D}_{\pi}$, we may assume that $\left.\mu_{\alpha}\right|_{\Lambda_{\psi}\left(D_{x}\right)} \cong \pi$. In this case, we may choose a basis $\left\{v_{i}\right\}$ of $D_{x}$ such that

$$
\mu_{\alpha}(\omega) \Lambda_{\psi}\left(v_{j}\right)=\sum_{i=1}^{d} \omega\left(V(\pi)_{i, j}\right) \Lambda_{\psi}\left(v_{i}\right)
$$

This, together with (A.1), implies that $\left(\operatorname{id}_{\mathscr{P}} \otimes \omega\right) \circ \alpha\left(v_{j}\right)=\sum_{i=1}^{d} \omega\left(V(\pi)_{i, j}\right) v_{i}$, which is in turn equivalent to

$$
\alpha\left(v_{j}\right)=\sum_{i=1}^{d} v_{i} \otimes V(\pi)_{i, j} .
$$

Hence $D_{x}$ is an $\alpha$-invariant subspace of $\mathscr{P}$ such that $\pi_{D}$ is equivalent to $\pi$. Therefore, $x \in \mathscr{D}_{\pi}$ as claimed.

We summarize, as a proposition, the results of the preceding discussion.

Proposition A.1. For each irreducible representation $\pi$, the $\pi$-spectral subspace $\mathscr{P}_{\pi}$ with respect to the action $\alpha$ coincides with the subspace generated by the $\alpha$-invariant subspaces $D$ of $\mathscr{P}$ with the property that $\pi_{D}$ is equivalent to $\pi$.

Part B. Remarks on the actions $\bar{\alpha}$ and $\tilde{\alpha}$ This subsection is concerned with some relations among the actions $\alpha, \bar{\alpha}$ and $\tilde{\alpha}$. In what follows, $\mathrm{K}$ is a general (not necessarily compact) Kac algebra.

Proposition B.1. Suppose that $\mathscr{P}$ is properly infinite. Then there exists $a *-$ isomorphism $\Psi$ from $\mathscr{P}$ onto $\mathscr{P} \otimes \bar{F}_{\infty}$ so that, through this $\Psi, \alpha$ is cocycle conjugate to the action $\bar{\alpha}$ defined in Section 1 , where $\mathscr{F}_{\infty}$ denotes the countably decomposable infinite factor of type $\mathrm{I}$.

Proof. By assumption, we may take a sequence $\left\{e_{n}\right\}_{n=1}^{\infty}$ of projections in $\mathscr{P}$ such that $e_{n} \sim 1$ and $\sum_{n=1}^{\infty} e_{n}=1$. Then, for each $n \in$, choose a partial isometry $v_{n} \in \mathscr{P}$ with $v_{n}^{*} v_{n}=e_{n}$ and $v_{n} v_{n}^{*}=1$. Let $\left\{u_{i, j}\right\}_{i, j=1}^{\infty}$ be a system of matrix units in $\mathscr{F}_{\infty}$. It is well-known that, with $x_{i, j}=v_{i}^{*} x v_{j}$, the equation

$$
\Psi(x)=\sum_{i, j=1}^{\infty} x_{i, j} \otimes u_{i, j} \quad(x \in \mathscr{P})
$$

defines a $*$-isomorphism $\Psi$ from $\mathscr{P}$ onto $\mathscr{P} \otimes \mathscr{F}_{\infty}$. We put $\beta=\left(\Psi \otimes \operatorname{id}_{\mathscr{M}}\right) \circ$ $\alpha \circ \Psi^{-1}$, which is, by definition, an action of $\mathrm{K}$ on $\mathscr{P} \bar{\otimes} \mathscr{F} \infty$. Next we define an element $U$ of $\mathscr{P} \otimes \bar{F}_{\infty} \bar{\otimes} \mathscr{M}$ by 
ON DOMINANCY OF MINIMAL ACTIONS OF COMPACT KAC ALGEBRAS... 317

$$
U=\sum_{n=1}^{\infty}\left(w_{n} \otimes 1\right) \beta\left(w_{n}^{*}\right),
$$

where $w_{n}=\pi\left(v_{n}\right)$. Then $U$ is a unitary. Indeed, we have

$$
\begin{aligned}
U^{*} U & =\sum_{n, m=1}^{\infty} \beta\left(w_{n}\right)\left(w_{n}^{*} \otimes 1\right)\left(w_{m} \otimes 1\right) \beta\left(w_{m}^{*}\right)=\sum_{n, m=1}^{\infty} \delta_{n, m} \beta\left(w_{n} w_{m}^{*}\right) \\
& =\sum_{n=1}^{\infty} \beta\left(w_{n} w_{n}^{*}\right)=1 .
\end{aligned}
$$

Similarly, we obtain $U U^{*}=1$. Moreover, on $\mathscr{P} \bar{\otimes} \mathscr{F}_{\infty} \bar{\otimes} \mathscr{M} \bar{\otimes} \mathscr{M}$, one has

$$
\begin{aligned}
U_{123}\left(\beta \otimes \operatorname{id}_{\mathscr{M}}\right)(U) & =\sum_{n, m=1}^{\infty}\left(w_{n} \otimes 1 \otimes 1\right)\left(\beta\left(w_{n}^{*}\right) \otimes 1\right)\left(\beta \otimes \mathrm{id}_{\mathscr{M}}\right)\left(\left(w_{m} \otimes 1\right) \beta\left(w_{m}^{*}\right)\right) \\
& =\sum_{n, m=1}^{\infty}\left(w_{n} \otimes 1 \otimes 1\right)\left(\beta\left(w_{n}^{*} w_{m}\right) \otimes 1\right)\left(\beta \otimes \mathrm{id}_{\mathscr{M}}\right)\left(\beta\left(w_{m}^{*}\right)\right) \\
& =\sum_{n=1}^{\infty}\left(w_{n} \otimes 1 \otimes 1\right)\left(\beta \otimes \operatorname{id}_{\mathscr{M}}\right)\left(\beta\left(w_{m}^{*}\right)\right) \\
& =\sum_{n=1}^{\infty}\left(w_{n} \otimes 1 \otimes 1\right)\left(\operatorname{id}_{\mathscr{P} \overline{\mathscr{F}}_{\infty}} \otimes \Gamma\right)\left(\beta\left(w_{n}^{*}\right)\right) \\
& =\left(\operatorname{id}_{\mathscr{P} \otimes} \mathscr{F}_{\infty} \otimes \Gamma\right)(U) .
\end{aligned}
$$

This shows that $U$ is a $\beta$-cocycle [E]. Since $w_{n}^{*}\left(1 \otimes u_{k, \ell}\right) w_{m}=\delta_{n, k} \delta_{\ell, m} \cdot 1$, we can easily verify

$$
U \beta\left(1 \otimes u_{k, \ell}\right) U^{*}=1 \otimes u_{k, \ell} \otimes 1=\bar{\alpha}\left(1 \otimes u_{k, \ell}\right)
$$

for any $k, \ell \in \mathrm{N}$. Meanwhile, from $w_{n}^{*}(x \otimes 1) w_{m}=\delta_{n, m} \Psi(x)$ for any $x \in \mathscr{P}$, it follows that

$$
\begin{aligned}
U \beta(x \otimes 1) U^{*} & =\sum_{n, m=1}^{\infty}\left(w_{n} \otimes 1\right) \beta\left(w_{n}^{*}(x \otimes 1) w_{m}\right)\left(w_{m}^{*} \otimes 1\right) \\
& =\sum_{n=1}^{\infty}\left(w_{n} \otimes 1\right)\left(\Psi \otimes \mathrm{id}_{\mathscr{M}}\right)(\alpha(x))\left(w_{n}^{*} \otimes 1\right) \\
& =\sum_{n=1}^{\infty}\left(w_{n} \otimes 1\right)\left(\Psi \otimes \operatorname{id}_{\mathscr{M}}\right)(\alpha(x))\left(w_{n}^{*} \otimes 1\right) .
\end{aligned}
$$

Note that $\sum_{n=1}^{\infty}\left(w_{n} \otimes 1\right)\left(\Psi \otimes \mathrm{id}_{\mathscr{M}}\right)(y \otimes a)\left(w_{n}^{*} \otimes 1\right)=y \otimes 1 \otimes a$ for any $y \in \mathscr{P}$ and $a \in \mathscr{M}$. This, together with the above computation, yields

$$
U \beta(x \otimes 1) U^{*}=\alpha(x)_{13}=\bar{\alpha}(x \otimes 1) .
$$


Thus we have proven that $\operatorname{Ad} U \circ \beta=\bar{\alpha}$. Namely, $\beta$ is cocycle conjugate to $\bar{\alpha}$. Therefore, $\alpha$ is cocycle conjugate to $\bar{\alpha}$ by construction.

Corollary B.2. With the notation in Proposition B.1, we now assume that the fixed-point algebra $\mathscr{P}^{\alpha}$ of $\alpha$ is properly infinite. Then $\alpha$ is conjugate to $\bar{\alpha}$ through the isomorphism $\Psi$.

Proof. In the proof of Proposition B.1, we can take $e_{n}$ and $v_{n}$ from the inside of $\mathscr{P}^{\alpha}$ by assumption. Then $w_{n}$ 's belong to the fixed-point algebra of $\beta$, so that the $\beta$-cocycle $U$ in this case is the identity operator. This proves the assertion.

Corollary B.3. Suppose that $\mathscr{P}$ is properly infinite. Consider the induced action $\tilde{\alpha}$ of $\mathrm{K}$ on $\mathscr{P} \otimes \mathscr{L}\left(L^{2}(\varphi)\right)$ defined in Section 1, i.e., $\tilde{\alpha}=\operatorname{Ad}(1 \otimes W(\hat{\mathrm{K}})) \circ \bar{\alpha}$. Then $\alpha$ is cocycle conjugate to $\tilde{\alpha}$.

Proof. Since the unitary $1 \otimes W(\hat{\mathrm{K}})$ is an $\bar{\alpha}$-cocycle by Théorème IV.3. (ii) of [ES2], the assertion follows from Proposition B.1.

\section{REFERENCES}

[AHKT] H. Araki, R. Haag, D. Kastler and M. Takesaki, Extension of KMS states and chemical potential, Comm. Math. Phys. 53 (1977), 97-134.

[C] A. Connes, Periodic automorphisms of the hyperfinite factor of type $\mathrm{II}_{1}$, Acta Sci. Math. 39 (1977), 39-66.

[CT] A. Connes and M. Takesaki, The flow of weights on factors of type III, Tohoku Math. J. 29 (1977), 473-575.

[DeC] J. De Cannière, On the intrinsic group of a Kac algebra, Proc. London Math. Soc. 39 (1979), 1-20.

[E] M. Enock, Produit croisé d'une algèbre de von Neumann par une algèbre de Kac, J. Funct. Anal. 26 (1977), 16-47.

[ES1] M. Enock and J.M. Schwartz, Kac Algebras and Duality of Locally Compact Groups, Springer-Verlag, Berlin Heidelberg 1992.

[ES2] M. Enock and J.M. Schwartz, Produit croisé d'une algèbre de von Neumann par une algèbre de Kac II, Publ. Res. Inst. Math. Sci. 16 (1980), 189-232.

[F] G.B. Folland, A Course in Abstract Harmonic Analysis. Studies in Adv. Math. CRC Press 1995.

[ILP] M. Izumi, R. Longo and S. Popa, A Galois correspondence for compact groups of automorphisms of von Neumann algebras with a generalization to Kac algebras, J. Funct. Anal. 155 (1998), 25-63.

[J1] V.F.R. Jones, Actions of finite groups on the hyperfinite type $\mathrm{II}_{1}$ factor, Mem. Amer. Math. Soc. 237 (1980).

[J2] V.F.R. Jones, Prime actions of compact abelian groups on the hyperfinite type $\mathrm{II}_{1}$ factor, J. Operator Theory 9 (1983), 181-186.

[JT] V.F.R. Jones and M. Takesaki, Actions of compact abelian groups on semifinite injective factors, Acta Math. 153 (1984), 213-258.

[KP] G.I. Kac and V.G. Paljutkin, Finite ring-groups, Transl. Moscow Math. Soc. (1966), 251-294. 
ON DOMINANCY OF MINIMAL ACTIONS OF COMPACT KAC ALGEBRAS... 319

[KT] Y. Kawahigashi and M. Takesaki, Compact abelian group actions on injective factors, J. Funct. Anal. 105 (1992), 112-128.

[N] Y. Nakagami, Some remarks on crossed products of von Neumann algebras by Kac algebras, Yokohama Math. J. 27 (1979), 141-162.

[NT] Y. Nakagami and M. Takesaki, Duality for crossed products of von Neumann algebras, Lecture Notes in Math. 731 (1979).

[OPT] D. Olesen, G.K. Pedersen and M. Takesaki, Ergodic actions of compact abelian groups, J. Operator Theory 3 (1980), 237-269.

[PW] S. Popa and A. Wassermann, Actions of compact Lie groups on von Neumann algebras, C.R. Acad. Sci. Paris 315 (1992), 421-426.

[R] J.E. Roberts, Cross products of von Neumann algebras by group duals, Sympos. Math. XX (1976).

[S] J.M. Schwartz, Sur la structure des algèbres de Kac I, J. Funct. Anal. 34 (1979), 370406.

[T] K. Thomsen, Compact abelian prime actions on von Neumann algebras, Trans. Amer. Math. Soc. 315 (1989), 255-272.

[TT] M. Takesaki and N. Tatsuuma, Duality and subgroups, Ann. of Math. 93 (1971), 344 364.

[W1] A. Wassermann, Ergodic actions of compact groups on operator algebras I. General theory, Ann. of Math. 130 (1989), 273-319.

[W2] A. Wassermann, Ergodic actions of compact groups on operator algebras II: Classification of full multiplicity ergodic actions, Canad. J. Math. XL (1988), 1482-1527.

[W3] A. Wassermann, Ergodic actions of compact groups on operator algebras III. Classification for SU(2), Invent. Math. 93 (1988), 309-355.

[W] S.L. Woronowicz, Compact matrix pseudogroups, Comm. Math. Phys. 111 (1987), 613665 .

DEPARTMENT OF MATHEMATICS

FACULTY OF SCIENCE

HOKKAIDO UNIVERSITY

SAPPORO 060

JAPAN

E-mail address: yamanouc@math.sci.hokudai.ac.jp 